\title{
Constraints on a light leptophobic mediator from LEP data
}

\section{Manuel Drees and Zhongyi Zhang}

Bethe Center for Theoretical Physics, Bonn University, 53115 Bonn, Germany

E-mail: drees@th.physik.uni-bonn.de, zhongyi@th.physik.uni-bonn.de

ABSTRACT: We apply data taken at the $e^{+} e^{-}$collider LEP in the 1990's at center-of-mass energy up to $209 \mathrm{GeV}$ to constrain Dark Matter models with a light leptophobic spin-1 mediator $R$. We assume that the dark sector particle (DSP) is a spin $-1 / 2$ fermion $\chi$. This scenario is well studied in the context of LHC searches for mediator mass from $100 \mathrm{GeV}$ to several TeV. Emission of the mediator off a quark or antiquark at LEP gives rise to di-jet plus missing energy and 4 -jet signatures, which we use to limit the relevant couplings. We focus on scenarios with $2 m_{\chi}>m_{R}$, which are poorly constrained by LHC data. We recast published searches by the ALEPH collaboration. For $m_{\chi} \lesssim 20 \mathrm{GeV}$ the best bounds result from an analysis at $\sqrt{s} \simeq M_{Z}$ of di-jet plus missing energy events. For heavier DSP but $m_{R} \lesssim 70 \mathrm{GeV}$ meaningful bounds can be derived from a four jet analysis at $\sqrt{s}=183 \mathrm{GeV}$. Unfortunately published searches using four jet final states at $\sqrt{s} \simeq M_{Z}$ use only a small fraction of the total data sample. Moreover, all published searches for di-jet plus missing energy final states at $\sqrt{s} \geq 130 \mathrm{GeV}$ have poor efficiency for our model; we therefore design new cuts that combine good background rejection with higher efficiency. Re-analyzing the higher energy data using our new cuts, and an analysis of the complete four jet data sample taken at $\sqrt{s} \simeq M_{Z}$, can explore new regions of parameter space.

KEYwords: Dark matter, e+-e- Experiments, Jets, Beyond Standard Model

ARXIV EPRINT: 1805.02780 


\section{Contents}

1 Introduction 1

2 The simplified model $\quad 3$

2.1 Lagrangian and free parameters 3

2.2 Dark matter constraints 4

$\begin{array}{lll}2.3 & \text { Perturbativity condition } & 6\end{array}$

2.4 Unitarity condition 7

2.5 Summary: free parameters of the model $\quad 7$

3 Application of LEP data $\quad 8$

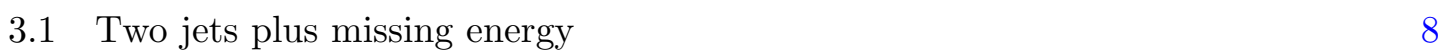

3.1.1 Analysis of LEP2 data 8

3.1.2 Analysis of LEP1 data 14

$\begin{array}{lll}3.2 & \text { Four jet analysis } & 16\end{array}$

4 Improved analysis of LEP2 data $\quad 19$

5 Summary and conclusions $\quad 24$

\section{Introduction}

The Standard Model (SM) of particle physics lacks an adequate candidate for dark matter [1]. Particle physics explanations of dark matter therefore typically include a "dark sector" containing (at least) one stable dark matter candidate (called DSP for Dark Sector Particle in this paper), and at least one mediator coupling the DSP to SM particles. In principle this mediator could be the well-known $Z$ or $125 \mathrm{GeV}$ Higgs boson, but these "portal" models are by now very tightly constrained [2-4]. Here we are interested in models where the mediator is not part of the SM. Frequently it is a massive scalar or vector boson. Therefore, a simplified model approach [5-7] allows to constrain many UV complete extensions of the SM. Simplified models usually have a relatively small number of free parameters, allowing exhaustive scans of the parameter space. Models designed to describe the scattering of DSPs on ordinary matter, as in "direct search" experiments, have to specify the couplings of the mediator(s) to hadrons and to the DSP. This suffices to fix the rate of monojet (and similar) events at the LHC. Since no excess of such events has been found, LHC data have given strong constraints for mediator masses below about $1 \mathrm{TeV}$ that can decay invisibly, e.g. into a pair of dark matter particles [8, 9]. Moreover, mediator masses roughly between 1 and $2.5 \mathrm{TeV}$ are also constrained by searches for dijet final states $[10,11]$. Very recently this range has been extended downward by using 
special search strategies [12]; preliminary results using events with a hard third jet recoiling against a "fat jet" allowed CMS to extend the search range down to $50 \mathrm{GeV}$ [13], for coupling strength to (light) quarks $\gtrsim 0.2{ }^{1}$

It should be noted that more complete models are often subject to additional constraints. For example, $Z^{\prime}$ models based on extending the SM gauge group with an additional $\mathrm{U}(1)$ factor were investigated in [14-19], and supersymmetric models in [20-22]. However, many or most of these constraints are not directly related to the dark matter problem.

Although the Large Electron Positron collider (LEP) at CERN ceased operations nearly twenty years ago, and only covered center-of-mass (cms) energies up to $209 \mathrm{GeV}$, the cleaner environment and the distinct energy range still offer some advantages for certain regions of parameter space. In this work we focus on a simplified model with a vector mediator $R$ coupling to the DSP and quarks. We use the framework of ref. [23], which starts from the very general assumption that the new mediator couples to all different kinds of SM particles, including gauge bosons, leptons and quarks. It uses LHC data (from run 1) in order to derive stringent upper bounds on many of these couplings. These constraints are quite strong if on-shell decays of the mediator to DSPs are possible, or if the mediator has sizable couplings to leptons. We saw above that (in some cases still preliminary) constraints from LHC searches for purely hadronic final states have become quite strong, if $m_{R}>50 \mathrm{GeV}$. However, the published constraints apply to couplings to first generation quarks, which are strongly constrained by direct dark matter searches. The annihilation cross section of the DSP $\chi$ into hadronic final states can therefore still be sufficiently large for $\chi$ to be a good thermal WIMP (Weakly Interacting Massive Particle) candidate [24] in standard cosmology. In this study we also focus on the $\bar{\chi} \chi R$ and $\bar{q} q R$ couplings. Note that together with the masses $m_{R}$ and $m_{\chi}$ these are the key parameters determining both the direct WIMP detection rate and (if the other couplings are small) the relic density.

There are also purely theoretical constraints on the model. Ref. [23] derived an upper bound on the couplings from the requirement that perturbation theory can be applied, since we do not know how to constrain these couplings otherwise. Moreover, as pointed out in ref. [25], if $R$ has non-vanishing axial vector couplings to $\chi$, unitarity imposes an upper bound on the ratio of DSP and mediator masses. We apply the same perturbativity and unitarity conditions in the part of parameter space that could have been probed by LEP experiments.

LHC data only probe configurations where the mediator is essentially on-shell. In contrast, in this study, which focuses on a light mediator, we consider cases where the DSP pair can only be generated through off-shell processes. We notice an enhancement of the cross section if the mediator has an axial vector coupling to $b$-quarks and $m_{R}<$ $m_{b}$ because the longitudinal part of the mediator contributes a term $\propto m_{b}^{2} / m_{R}^{2}$ to the four-jet cross section. Similarly, in the presence of an axial vector coupling to the DSP the di-jet plus missing energy cross section may increase with increasing $m_{\chi}$, contrary to naive expectations. However, the unitarity constraints imply that these terms cannot be arbitrarily large.

\footnotetext{
${ }^{1}$ This analysis also found a slight excess of events corresponding to a mediator mass of about $115 \mathrm{GeV}$.
} 
The remainder of this article is organized as follows. In section 2 the Lagrangian of the simplified model is introduced, and bounds on the relevant couplings from theoretical considerations and non-collider experiments are discussed. In section 3 we recast searches for di-jet plus missing energy and 4-jet final states performed by the ALEPH collaboration $[26,27]$. We discuss the bounds resulting from these published searches and the cut efficiencies when applied to our model. In section 4 we introduce a set of specially designed cuts for the di-jet plus missing energy signature that have much higher efficiency for our signal than the published searches. Although we do not include the detector simulation in the test of the background suppression, the result still shows the potential of the LEP data to improve on the bounds derived in section 3. Finally, section 5 is devoted to a summary and some conclusions.

\section{The simplified model}

In this section we first describe the Lagrangian of the simplified model we consider. We then discuss limits on the model parameters that follow if the DSP is assumed to be a thermal WIMP, which is subject to stringent constraints from direct dark matter search experiments. In the following two subsections we discuss upper bounds on the couplings that result from perturbativity and unitarity constraints. In the final subsection the precollider bounds on the remaining free parameters are summarized and our final choice of free parameters is discussed.

\subsection{Lagrangian and free parameters}

As discussed in the Introduction, we consider a simplified model [23] where a massive spin-1 mediator connects the DSP to SM particles. The Lagrangian can then be written as

$$
\mathcal{L}=\mathcal{L}_{\mathrm{SM}}+\mathcal{L}_{\mathrm{DSP}}+\mathcal{L}_{R}+\mathcal{L}_{I}
$$

We assume the DSP to be a spin-1/2 Dirac fermion. A Majorana fermion cannot have a vector interaction, but is otherwise basically the same as a Dirac fermion for our purposes. ${ }^{2}$ The DSP part of the Lagrangian is therefore:

$$
\mathcal{L}_{\mathrm{DSP}}=\bar{\chi}\left(i \not \partial-m_{\chi}\right) \chi
$$

In MadGraph convention [28] the mediator part of the Lagrangian is:

$$
\mathcal{L}_{R}=-\frac{1}{4} F^{\mu \nu} F_{\mu \nu}-\frac{1}{2} m_{R}^{2} R^{\mu} R_{\mu}, \quad \text { with } F_{\mu \nu} \equiv \partial_{\mu} R_{\nu}-\partial_{\nu} R_{\mu} .
$$

Finally, the interactions of the mediator with fermions are described by the Lagrangian

$$
\mathcal{L}_{I}=\sum_{q} R_{\mu} \bar{q} \gamma^{\mu}\left(g_{q}^{V}-g_{q}^{A} \gamma^{5}\right) q+R_{\mu} \bar{\chi} \gamma^{\mu}\left(g_{\chi}^{V}-g_{\chi}^{A} \gamma^{5}\right) \chi .
$$

\footnotetext{
${ }^{2} \mathrm{~A}$ complex scalar DSP behaves similar to a Majorana DSP if $m_{R}>m_{b}, m_{\chi}$. However, the contribution from the exchange of longitudinal messenger particles vanishes identically in this case, i.e. there are no terms that are enhanced by $m_{b} m_{\chi} / m_{R}^{2}$.
} 
The free parameters of our model are thus the mediator mass $m_{R}$, the DSP mass $m_{\chi}$, and the couplings of the mediator to quarks $\left(g_{q}^{V}, g_{q}^{A}\right)$ and to the $\operatorname{DSP}\left(g_{\chi}^{V}, g_{\chi}^{A}\right)$. In total, there are 16 parameters. However, since this study uses data from $e^{+} e^{-}$collision up to $\sqrt{s}=209 \mathrm{GeV}$, top quarks cannot contribute to the final state. Therefore the couplings $g_{t}^{V}$ and $g_{t}^{A}$ are irrelevant, so that 14 relevant free parameters remain.

An exhaustive scan of a 14-dimensional parameter space is not feasible with our computational resource. However, as we will see in the following subsections, non-collider constraints force many of these couplings to be very small, so that we can set them to zero for our purposes.

\subsection{Dark matter constraints}

In the standard thermal WIMP scenario, the dark matter relic density is essentially inversely proportional to the total DSP annihilation cross section computed in the nonrelativistic limit [24]. In our model the DSP can always annihilate into sufficiently light quarks, with cross section [29]:

$$
\begin{aligned}
v \sigma(\bar{\chi} \chi & \rightarrow \bar{q} q) \simeq \frac{3 m_{\chi}^{2}}{2 \pi\left(m_{R}^{2}-4 m_{\chi}^{2}\right)^{2}} \sqrt{1-\frac{m_{q}^{2}}{m_{\chi}^{2}}} \\
\cdot & {\left[\left(g_{q}^{V}\right)^{2}\left(g_{\chi}^{V}\right)^{2}\left(2+\frac{m_{q}^{2}}{m_{\chi}^{2}}\right)+2\left(g_{q}^{A}\right)^{2}\left(g_{\chi}^{V}\right)^{2}\left(1-\frac{m_{q}^{2}}{m_{\chi}^{2}}\right)+\left(g_{q}^{A}\right)^{2}\left(g_{\chi}^{A}\right)^{2} \frac{m_{q}^{2}}{m_{\chi}^{2}} \frac{\left(4 m_{\chi}^{2}-m_{R}^{2}\right)^{2}}{m_{R}^{4}}\right] . }
\end{aligned}
$$

Here $v$ is the relative velocity between $\chi$ and $\bar{\chi}$. The last term on the right-hand side (rhs) of eq. (2.5) is due to the exchange of longitudinal $R$-bosons. Note that is enhanced $\propto m_{\chi}^{2} m_{q}^{2} / m_{R}^{4}$ for small mediator masses; at the same time it is suppressed $\propto m_{q}^{2} / m_{\chi}^{2}$ if $m_{R}>2 m_{\chi} \gg m_{q}$. The numerator of this term implies that it does not have a pole at $s \simeq 4 m_{\chi}^{2}=m_{R}^{2}$. If the vectorial couplings do not vanish, this term is therefore only relevant if the exchanged mediator is quite far off-shell. Notice also that this term is proportional to the product of axial vector couplings, i.e. it is absent for a purely vectorial theory. At the same time it is the only term that survives for vanishing vector couplings, e.g. if $\chi$ is a Majorana particle.

Moreover, for $m_{\chi}>m_{R}$ a $\chi \bar{\chi}$ pair can also annihilate into two mediators, which subsequently decay to quarks. The corresponding cross section is [29]:

$$
\begin{aligned}
v \sigma(\bar{\chi} \chi \rightarrow R R)= & \frac{\left(m_{\chi}^{2}-m_{R}^{2}\right)^{3 / 2}}{4 \pi m_{\chi}\left(m_{R}^{2}-2 m_{\chi}^{2}\right)^{2}} \\
& \cdot\left\{8\left(g_{\chi}^{A}\right)^{2}\left(g_{\chi}^{V}\right)^{2} \frac{m_{\chi}^{2}}{m_{R}^{2}}+\left[\left(g_{\chi}^{A}\right)^{4}-6\left(g_{\chi}^{A}\right)^{2}\left(g_{\chi}^{V}\right)^{2}+\left(g_{\chi}^{V}\right)^{4}\right]\right\} .
\end{aligned}
$$

The first term in the second line again gives an enhancement $\propto m_{\chi}^{2} / m_{R}^{2}$. Note that in the limit $v \rightarrow 0$, which we applied here, the contribution $\propto\left(g_{\chi}^{A}\right)^{4} / m_{R}^{4}$, which is due to the production of two longitudinal $R$ bosons, vanishes. Moreover, the cross section (2.6) is quite strongly phase space suppressed near threshold where $m_{\chi} \simeq m_{R}$.

Since the predicted DSP relic density is inversely proportional to the total $\chi \bar{\chi}$ annihilation cross section, requiring that the predicted DSP density is not larger than the 
total observed dark matter density imposes a lower bound on (sums of products of) the relevant couplings if the masses are fixed. The detailed analysis of ref. [29] shows that for $m_{R} \leq 100 \mathrm{GeV}$ this bound is easily satisfied if all axial vector couplings are $\gtrsim 0.3$ even for vanishing vector couplings. We will see below that LEP data only allow to probe significantly smaller $m_{R}$. We confirm that for coupling strengths of interest to LEP physics, in standard cosmology the thermal DSP relic density is always much below the desired dark matter density, unless the DSP is very light (with $m_{\chi}<m_{R}$ so that $\chi \bar{\chi} \rightarrow R R$ annihilation is suppressed) and has very small couplings to light quarks (see below).

The signal in direct dark matter detection experiments depends essentially on the mass of the dark matter particle and its scattering cross section on nucleons. For the latter one usually distinguishes between spin-dependent (SD) and spin-independent (SI) contributions. The corresponding cross sections can be written as [29]:

$$
\sigma_{N}^{\mathrm{SD}}=a_{N}^{2} \frac{3 \mu_{N}^{2}}{\pi m_{R}^{4}} ; \quad \sigma_{N}^{\mathrm{SI}}=f_{N}^{2} \frac{3 \mu_{N}^{2}}{\pi m_{R}^{4}} .
$$

Here $N=n, p$ and

$$
\mu_{N}=\frac{m_{\chi} m_{N}}{m_{\chi}+m_{N}}
$$

is the reduced mass of the DSP-nucleon system. The coefficients $f_{N}$ appearing in $\sigma_{N}^{\mathrm{SI}}$ are simply given by products of couplings:

$$
f_{p}=g_{\chi}^{V}\left(2 g_{u}^{V}+g_{d}^{V}\right) ; f_{n}=g_{\chi}^{V}\left(g_{u}^{V}+2 g_{d}^{V}\right)
$$

where the differences are due to the different valence quark content of neutrons and protons. Note that sea quarks do not contribute, since quarks and antiquarks couple with opposite sign to $R$; their contributions cancel, since here the coherent coupling to the entire nucleon (in fact, in most cases to an entire nucleus) is relevant. Finally, the coefficients $a_{N}$ appearing in $\sigma_{N}^{\mathrm{SD}}$ are:

$$
a_{N}=g_{\chi}^{A} \sum_{q=u, d, s} \Delta q^{(N)} g_{q}^{A}
$$

Here $\Delta q^{(N)}$ is the contribution of the spin of quark $q$ to the total spin of nucleon $N$. They can be determined from polarized deep-inelastic scattering experiments. The current Particle Data Group values [30] are:

$$
\begin{aligned}
& \Delta u^{(p)}=\Delta d^{(n)}=0.84 \pm 0.02 ; \\
& \Delta u^{(n)}=\Delta d^{(p)}=-0.43 \pm 0.02 ; \\
& \Delta s^{(p)}=\Delta s^{(n)}=-0.09 \pm 0.02 .
\end{aligned}
$$

There are strong upper bounds on the spin-independent scattering cross section on the proton. For $m_{\chi} \gtrsim 5 \mathrm{GeV}$ the tightest constraint comes from the PandaX-II [31] experiment, whereas CRESST [32] data impose significant constraints for $m_{\chi} \gtrsim 0.5 \mathrm{GeV}$. We will see below that LEP data can only probe scenarios with $m_{R}<100 \mathrm{GeV}$. These bounds require 
$g_{u, d}^{V}$ to be below 0.1 , usually much below this value. Such small couplings have little influence on LEP physics, so we set $g_{u}^{V}=g_{d}^{V}=0 .^{3}$

The upper bounds on the spin-dependent cross sections become quite weak for WIMP mass below $4 \mathrm{GeV}$, but the bound on $\sigma_{n}^{\mathrm{SD}}$ is still below $10^{-2} \mathrm{pb}$ for $m_{\chi}=5 \mathrm{GeV}$ [33]. If $m_{R} \leq 10 \mathrm{GeV}$ this constraint suffices to imply $g_{u, d}^{A} \leq 0.1$, the bound on $g_{s}^{A}$ being somewhat weaker but still strong enough to force these couplings to be negligible for LEP physics. On the other hand, for $m_{R} \geq 50 \mathrm{GeV} \mathcal{O}(1)$ axial vector couplings are allowed even for the light quarks if we scale the bound on the scattering cross section by the ratio of the predicted $\chi$ relic density and the total observed dark matter density. However, in that case $\chi$ does not make a good thermal dark matter candidate. In most scenarios where the predicted $\chi$ relic density in standard cosmology is at least a sizable fraction of the observed dark matter density the upper bound on the spin dependent cross section for $m_{\chi} \gtrsim 4 \mathrm{GeV}$ requires the axial vector couplings to be too small to significantly affect LEP cross section. We therefore set $g_{u}^{A}=g_{d}^{A}=g_{s}^{A}=g_{c}^{A}=0$; we require vanishing axial vector coupling to charm quarks since strange and charm quarks reside in the same $\mathrm{SU}(2)$ doublet.

We are then left with eight free parameters: four couplings of $R$ to quarks, two couplings of $R$ to the DSP, and the masses of $R$ and the DSP.

\subsection{Perturbativity condition}

We will use leading order perturbation theory to derive constraints on our model from published LEP data. Perturbation theory becomes unreliable when the couplings become too large. Our calculations depend on the SM electroweak couplings, which are perturbative, and on the couplings of the mediator $R$. We constrain the latter through the simple condition

$$
\Gamma_{R}<m_{R},
$$

where $\Gamma_{R}$ is the total decay width of $R . \quad R$ can decay into $q \bar{q}$ and $\chi \bar{\chi}$ pairs, with partial widths:

$$
\begin{aligned}
\Gamma(R \rightarrow q \bar{q}) & =\frac{m_{R}}{4 \pi} \sqrt{1-4 z_{q}}\left[\left(g_{q}^{V}\right)^{2}+\left(g_{q}^{A}\right)^{2}+z_{q}\left(2\left(g_{q}^{V}\right)^{2}-4\left(g_{q}^{A}\right)^{2}\right)\right] \\
\Gamma(R \rightarrow \chi \bar{\chi}) & =\frac{m_{R}}{12 \pi} \sqrt{1-4 z_{\chi}}\left[\left(g_{\chi}^{V}\right)^{2}+\left(g_{\chi}^{A}\right)^{2}+z_{\chi}\left(2\left(g_{\chi}^{V}\right)^{2}-4\left(g_{\chi}^{A}\right)^{2}\right)\right] .
\end{aligned}
$$

Here $z_{f} \equiv m_{f}^{2} / m_{R}^{2}$. The factor of 3 in the first equation comes from the colors of quarks. Of course, these widths are nonzero only for $m_{R}>2 m_{f}$, i.e. $z_{F}<0.25$. The perturbativity condition can thus be written as

$$
\sum_{2 m_{f}<m_{R}} N_{f} \sqrt{1-4 z_{f}}\left[\left(g_{f}^{V}\right)^{2}+\left(g_{f}^{A}\right)^{2}+z_{f}\left(2\left(g_{f}^{V}\right)^{2}-4\left(g_{f}^{A}\right)^{2}\right)\right]<12 \pi
$$

\footnotetext{
${ }^{3}$ The bounds on the spin-independent cross section have been derived under the assumption of equal scattering cross section on neutrons and protons, which need not be the case in our scenario. In fact, the cross section for scattering on any one isotope can be made to vanish for a particular (negative) ratio of $g_{u}^{V} / g_{d}^{V}$. However, by now experiments using many different isotopes have been performed, allowing to constrain $g_{u}^{V}$ and $g_{d}^{V}$ separately.
} 
This constraint can be used for $m_{R} \geq 1 \mathrm{GeV}$, so that at least decays into strange quarks are possible. For somewhat heavier mediators, which can also decay into $c \bar{c}$ and perhaps $\chi \bar{\chi}$ pairs, the constraint (2.14) becomes stronger. We will only use combinations of parameters that respect this bound.

\subsection{Unitarity condition}

Another important kind of constraint has first been discussed in ref. [25]: unitarity limits the size of the axial vector couplings of fermions $f$ to the mediator $R$. One way to see this is to consider the cross section for $f \bar{f} \rightarrow R_{L} R_{L}$, where $R_{L}$ denotes a longitudinally polarized $R$ boson. For fixed (nonzero) relative velocity between $f$ and $\bar{f}$, the matrix element scales like $\left(g_{f}^{A} m_{f} / m_{R}\right)^{2}$. This violates unitarity, unless

$$
g_{f}^{A} \frac{m_{f}}{m_{R}} \leqslant \sqrt{\frac{\pi}{2}} .
$$

Note that this bound applies both to the DSP, $f=\chi$, and to the quarks with non-vanishing axial vector coupling, $f=q$.

Another derivation of the unitarity constraint starts from the observation that in a renormalizable theory, $R$ must be a gauge boson. If fermion $f$ has non-vanishing axial coupling $g_{f}^{A}$ to $R$, the two-component fermions $f_{L}$ and $f_{R}$ must transform differently under the $R$ gauge symmetry. This implies that the (Dirac) mass term $m_{f} \overline{f_{L}} f_{R}$ is not invariant under the $R$ gauge symmetry. Hence $m_{f}$ must be due to the vacuum expectation value of some Higgs field that carries $R$ charge. The upper bound (2.15) then follows from the upper bound on the Yukawa coupling that gives rise to $m_{f}$.

The bound (2.15) limits the size of the enhancement due to the exchange of longitudinal $R$-bosons with axial vector coupling to massive fermions; see the discussion of eq. (2.5) above. We will see below that similar terms also appear in our signal cross sections. Neglecting the unitarity constraint (2.15) could thus lead to overly optimistic conclusions regarding the sensitivity of collider data to our model.

\subsection{Summary: free parameters of the model}

The perturbativity condition (2.14) is quite weak. The unitarity constraint (2.15) can be strong for small $m_{R}$, but only applies to the axial vector couplings, and in any case still allows non-negligible couplings. These constraints therefore do not reduce the number of free parameters, i.e. we still have the eight free parameters enumerated at the end of section 2.2. This parameter space is still too large for a thorough exploration.

We therefore assume equal vector couplings of $s, c$ and $b$ quarks. Recall that we set the vector couplings of $u$ and $d$ quarks to zero in order to satisfy constraints from direct detection experiments. As mentioned in the Introduction, we will investigate final states with either two jets and two DSPs, or with four jets. The searches we will use to probe $q \bar{q} \chi \bar{\chi}$ production do not require any flavor tagging, so to good approximation this cross section only depends on the sum $\left(g_{s}^{V}\right)^{2}+\left(g_{c}^{V}\right)^{2}+\left(g_{b}^{V}\right)^{2}$. Results for different ratios of the vector couplings therefore can be derived by simply re-scaling the results presented below. In contrast, the best published probe of the four-jet final state requires the detection of 
at least two $b$ (anti)quarks in the final state. Since $b \bar{b} b \bar{b}$ final states have a significantly higher probability of satisfying this requirements than final states with only one $b \bar{b}$ pair, $g_{b}^{V}$ contributes with higher weight to the final cross section after cuts than $g_{c}^{V}$ and $g_{s}^{V}$.

Recall that scenarios where a light $R$ can decay into a $\chi \bar{\chi}$ pair are strongly constrained by LHC "monojet" data. We will thus assume $m_{R}<2 m_{\chi}$. In that case the (tree-level) cross section for the four-jet final state is completely independent of the couplings $g_{\chi}^{V}$ and $g_{\chi}^{A}$. Moreover, the cross section for $q \bar{q} \chi \bar{\chi}$ production is then proportional to the product $\left(g_{q} g_{\chi}\right)^{2}$. It is thus sufficient to present results for a fixed ratio of the couplings of the mediator to quarks and to DSPs; results for different ratios can then be obtained by rescaling our results presented below.

In the end we are left with four free parameters: $m_{R}, m_{\chi}, g_{b}^{A}$ and $g_{q}^{V}$.

\section{Application of LEP data}

In this section we check whether published analyses of LEP data can impose significant constraints on the parameters of our model. We focus on analyses by the ALEPH collaboration $[26,27]$, because they are based on well-defined, and clearly described, cuts defining final states that receive contributions from the two processes we wish to probe. We expect data from the other three LEP experiments (DELPHI, L3 and OPAL) to have similar sensitivity, so a combined analysis could lead to somewhat stronger bounds.

In our numerical analysis we use FeynRules [34] to generate a model file in UFO format [35], MadGraph [28] to simulate the $e^{+} e^{-}$collision, and Pythia 8.2 [36] to perform the hadronization. We apply the cuts defining the relevant ALEPH analyses at the hadron level, neglecting detector resolution effects. In the following two subsections we discuss two-jet plus missing energy and four-jet final states, respectively.

\subsection{Two jets plus missing energy}

We start with the topology

$$
e^{+} e^{-} \rightarrow j e t+j e t+\not p,
$$

where $\not p$ stands for missing energy and momentum in the final state, i.e. the invariant mass of the two-jet system is significantly smaller than the center-of-mass energy $\sqrt{s}$. The extra Feynman diagrams contributing to this topology in our model are shown in figure 1. As usual we neglect the Higgs exchange diagrams since the $e^{+} e^{-} H$ coupling is tiny. Since the couplings, $g_{q}$ and $g_{\chi}$, appear together in figure 1, the experiment data bound the product of $g_{q}$ and $g_{\chi}$. Therefore, in the following section, bounds on $\sqrt{g_{q} g_{\chi}}$ are shown.

\subsubsection{Analysis of LEP2 data}

During the LEP2 period (data taken between 1995 and 2000, at center of mass energy $161 \mathrm{GeV} \leq \sqrt{s} \leq 209 \mathrm{GeV}$ ) ALEPH performed most searches for the topology (3.1) in the context of supersymmetric extensions of the SM. This includes searches for the pair production of squarks [37, 38] and neutralinos [39-43]. In addition, ALEPH searched for the production of an invisibly decaying Higgs boson produced in association with an on-shell $Z$ boson [44-46]. Each of these searches uses dedicated cuts to suppress the SM background. 

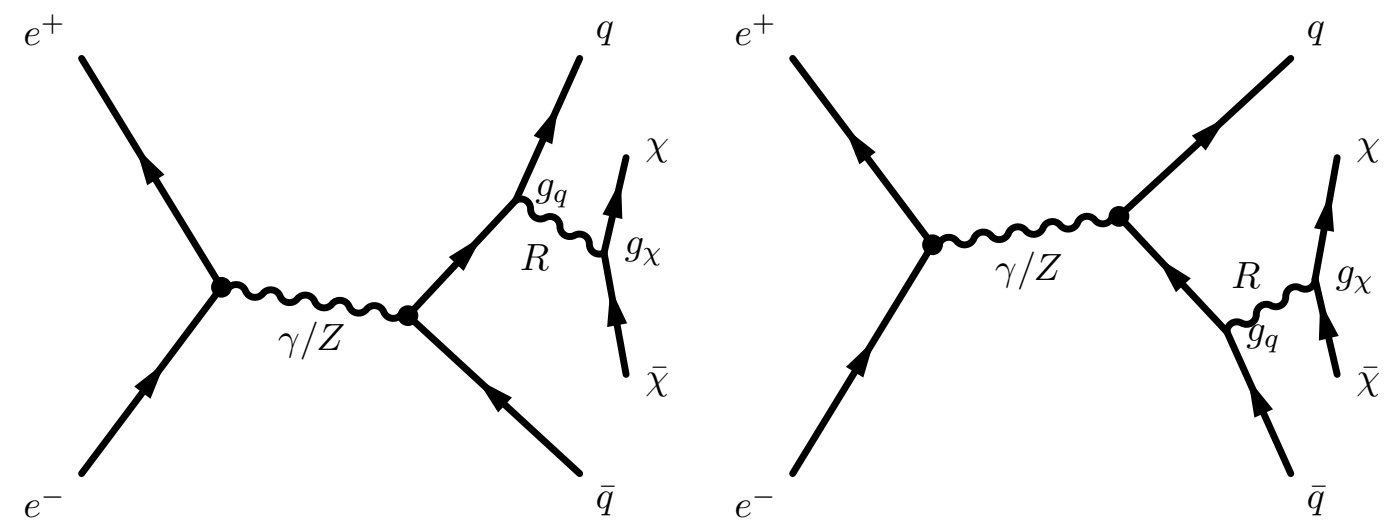

Figure 1. Leading order diagrams contributing to the final state (3.1) in our model. Note that the mediator $R$ is always off-shell in the region of parameter space we are interested in.

We generally find that the data taken at higher energies have better sensitivity to our model, if the event selection cuts are more or less independent of $\sqrt{s}$. The cross section for the four-body final state we are interested in depends quite sensitively on the available phase space. Note also that the integrated luminosity was higher at the higher energies. The total sensitivity is then essentially determined by the data taken at higher energy. On the contrary, if the cuts strongly depend on $\sqrt{s}$, the cut efficiencies may vary strongly; in this case one should consider all analyses together.

The neutralino searches fall in the second category. The analyses of the data taken at $\sqrt{s}=161$ and $172 \mathrm{GeV}$ [39] use quite different cuts than the analyses of the data taken at $\sqrt{s} \geq 183 \mathrm{GeV}$ [40-43]. At these higher energies, on-shell production of two $Z$ bosons becomes possible. The high-energy analyses impose a strong cut on the missing mass, which is designed to remove the $Z \bar{\nu} \nu$ background. Unfortunately this cut by itself excludes more than $90 \%$ of our signal, leading to a total cut efficiency of only about $2 \%$. On the other hand, the lower energy analyses use a cut on the visible mass, not on the missing mass, ${ }^{4}$ leading to a total cut efficiency of about $20 \%$ for our signal. The overall cross sections times luminosity at $\sqrt{s}=161$ and $172 \mathrm{GeV}$ are, however, too small. We therefore find that the analyses do not lead to significant bounds on our model.

For the invisibly decaying Higgs search, cut-based analyses were published only for data with $\sqrt{s} \leq 183 \mathrm{GeV}$ [44]. There is a published search for this channel using data taken at $\sqrt{s}=189 \mathrm{GeV}$ [46], but it uses a Neural Network; since we cannot reproduce this analysis, we cannot use it to constrain our model. ALEPH did not publish any search for an invisibly decaying Higgs using data taken at $\sqrt{s}>189 \mathrm{GeV}$. When applied to our signal, the cuts used in the analyses $[44,45]$ at $\sqrt{s}$ between 161 and $183 \mathrm{GeV}$ have an efficiency of less than $10 \%$. In this case the most harmful cuts are those related to the thrust and the reconstruction of the two jets. The relatively small cross sections, low integrated luminosity and insufficient cut efficiencies again imply that no meaningful constraints on our model can be derived.

\footnotetext{
${ }^{4}$ Note that in general there is no simple relation between the missing and the visible mass of a given event. The visible mass is defined as $M_{\mathrm{vis}}^{2}=P_{\mathrm{vis}}^{2}$, where $P_{\mathrm{vis}}$ denotes the sum of the $4-$ momenta of all "visible" particles; only neutrinos and DSPs are counted as "invisible". The missing mass is defined by $M_{\text {miss }}^{2}=\left(P_{\text {init }}-P_{\text {vis }}\right)^{2}$, where $P_{\text {init }}$ is the $4-$ momentum of the initial state. In some kinematical configurations both the visible and the missing mass are small.
} 

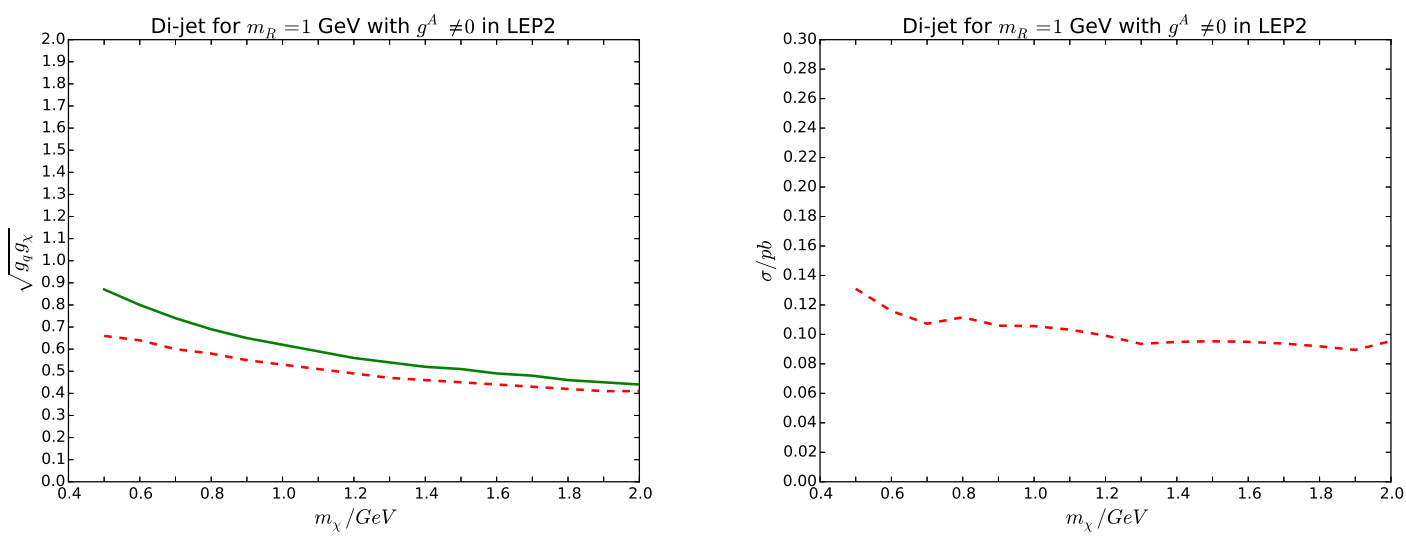

Figure 2. The left frame depicts the bound on $\sqrt{g_{q} g_{\chi}}$. The solid (green) curve shows the bound on $g^{A}$ from the unitarity condition (2.15). The dashed (red) curve shows the bound on $g^{A}$ from the combination of the unitarity condition and our recasting of the ALEPH squark pair search limits. The right frame shows the upper bound on the total signal cross section at $\sqrt{s}=208 \mathrm{GeV}$ from our recasting of the ALEPH limits. In these figures the mass of the mediator $m_{R}=1 \mathrm{GeV}$. Here all vector couplings have been set to zero, i.e. $g_{q}=g_{q}^{A}, g_{\chi}=g_{\chi}^{A}$.

We find the best sensitivity to our model when applying the cuts optimized for searches for squark pair production. Here cut-based analyses were published for the entire data set, including the highest energies. The cuts have been listed in section 7 of ref. [38]; ${ }^{5}$ when applied to our model, they frequently lead to an efficiency of $\geq 10 \%$. This is still not ideal, but sufficient to derive some meaningful constraints on the parameters of our model.

In this study we focus on the part of parameter space where on-shell $R \rightarrow \chi \bar{\chi}$ decays are not allowed, i.e. $m_{\chi}>m_{R} / 2$, since otherwise "monojet" searches by the LHC experiments $[29,47,48]$ give much tighter constraints. In this part of parameter space our signal process is a genuine $2 \rightarrow 4$ reaction, with rather low cross section. We find that our recasting of the ALEPH squark searches does not lead to significant constraints if $m_{R} \gtrsim 10 \mathrm{GeV}$. In figures 2 and 3 we therefore show results for $m_{R}=1,2$ and $5 \mathrm{GeV}$, respectively, focusing on scenarios with rather light DSP, $m_{R} / 2 \leq m_{\chi} \leq 2 m_{R}$. We find that the bounds on vector couplings are not as strong as those on the axial vector couplings, and do not depend strongly on $m_{R}$. Therefore, for nonzero $g^{A}$ and small $m_{\chi}$ we set the vector couplings to zero and derive the upper bound on the axial vector coupling from the ALEPH data in figures 2 and 3, while we show results for $g^{A}=0$ separately in figure 4 . For $m_{\chi} \gtrsim 4 \mathrm{GeV}$

\footnotetext{
${ }^{5}$ The cuts for "intermediate $\Delta M$ " usually turned out to give the tightest constraints. The influential cuts are $N_{\mathrm{ch}}>11, M_{\mathrm{vis}}>15 \mathrm{GeV}, p_{T} / \sqrt{s}>4 \%, E_{\mathrm{vis}} / \sqrt{s}<70 \%, E_{12} / \sqrt{s}<0.5 \%, \cos \theta_{\text {miss }}>0.8, \cos \theta_{T}>0.8$, $\Phi_{\text {acop }}<176^{\circ}, \Phi_{T}<177^{\circ}, E_{\text {Wedge }} / \sqrt{s}<12.5 \%$, Thrust $<0.94, p_{T} / E_{\text {vis }}>12.5 \%, E_{\text {had }} / \sqrt{s}<55 \%$, $E_{\mathrm{NH}} / E_{\mathrm{vis}}<30 \%$, and $E_{l 1}^{30} / \sqrt{s}>1 \%$. Here $N_{\mathrm{ch}}$ is the number of good tracks (i.e., of charged particles); $M_{\mathrm{vis}}$ is the invariant mass of the visible system, $E_{\mathrm{vis}}$ is its energy and $p_{T}$ is the absolute value of its transverse momentum, which is the same as the absolute value of the missing $p_{T} ; \theta_{\text {miss }}$ is the polar angle of the missing $p$ vector; $\theta_{T}$ is the polar angle of the thrust axis; $\Phi_{\text {acop }}$ is the acoplanarity angle; $E_{\text {had }}$ is the total measured energy excluding the contribution of identified charged leptons; and $E_{\text {Wedge }}$ is the energy in a $30^{\circ}$ azimuthal wedge around the missing transverse momentum. We also use many of these variables in the optimized cuts presented in section 4 , e.g. $E_{12}, E_{\mathrm{NH}}, E_{l 1}^{30}, \Phi_{T}$, where their definition and physical significance are discussed.
} 
and $g^{V}=0$ the resulting bound on $g^{A}$ is weaker than the unitarity bound (2.15). In this case we set the axial vector coupling such that the unitarity bound is saturated, and derive the resulting upper limit on the vector coupling. This is the strongest possible constraint on the vector coupling that can be derived from our recasting of the ALEPH squark pair search. The larger sensitivity to the axial vector coupling again comes from contributions $\propto m_{q} m_{\chi} / m_{R}^{2}$ to the Feynman amplitude.

These terms dominate the cross section for $m_{R}=1 \mathrm{GeV}$ (figure 2). As a result, the bound on the coupling becomes stronger as the DSP mass is increased. Evidently the enhanced contribution from longitudinal $R$ exchange over-compensates the reduction of the phase space. For the entire range of $m_{\chi}$ shown the bound is stronger than the unitarity limit. Note that we show the bounds on $\sqrt{g_{\chi}^{A} g_{b}^{A}}$, because the unitarity limit due to $m_{\chi}$ is different compared to that due to $m_{b}$. Moreover, all vector couplings have been set to zero. Strictly speaking we would have to allow some coupling at least to $s$ quarks in order to allow $R$ to decay; however, vector couplings $\ll 1$ will not affect the bound on the axial vector coupling. On the other hand, $\mathcal{O}(1)$ vector couplings would lead to a slightly stronger upper bound on the axial vector coupling.

The results for $m_{R}=2 \mathrm{GeV}$ (figure 3, top row) are qualitatively rather similar, but the bound on the axial vector coupling is weaker by a factor of about 1.5. As a result, for $m_{\chi} \geq 3.6 \mathrm{GeV}$ the upper bound on $g_{\chi}^{A}$ is actually set by the unitarity constraint (2.15). At $m_{\chi}=4 \mathrm{GeV}$ a vector coupling as large as 0.89 has been turned on in order to saturate our recasting of the ALEPH bound, for axial vector coupling at the unitarity limit. This leads to a slight increase of the upper bound on the cross section, shown in the right frame, which otherwise is very similar to the case with $m_{R}=1 \mathrm{GeV}$. Since in both cases $m_{R}$ is much smaller than all other relevant energy scales in the problem, in particular much smaller than the missing energy required by the cuts, it is not surprising that the upper bound on the cross section does not depend on $m_{R}$.

On the other hand, for $m_{R}=5 \mathrm{GeV} \simeq m_{b}$ (figure 3 , bottom row) the enhancement due to the exchange of longitudinal $R$-bosons no longer suffices to over-compensate the reduced phase space when $m_{\chi}$ is increased. The ALEPH squark searches now permit quite large axial vector couplings even for $m_{\chi}$ near $m_{R} / 2$. For $m_{\chi} \geq 4.5 \mathrm{GeV}$ this bound again becomes weaker than the unitarity constraint (2.15). Even if we saturate this constraint, sizable vector couplings are allowed by the ALEPH data, as shown by the dotted (blue) curve. Note that our perturbativity bound (2.14) requires $g_{q}^{V} \leq 2.5$ for $m_{R}=5 \mathrm{GeV}$. Figure 3 shows that our recasting of the ALEPH squark search limits leads to stronger upper bounds on this coupling if $m_{\chi} \leq 10 \mathrm{GeV}$.

Having considered nonzero $g^{A}$, the bounds on $g^{V}$ for vanishing $g^{A}$ are shown in figure 4 . Evidently the constraints on $g^{V}$ are much weaker than those on $g^{A}$. Recall, however, that $g^{V}$ is not constrained by the unitarity condition. The upper bound on $g^{V}$ is therefore set by LEP2 data for $m_{\chi} \lesssim 30 \mathrm{GeV}$; at even larger DSP masses, the LEP2 bound becomes weaker than the perturbativity condition (2.14). Another noticeable property is that for $m_{\chi}>10 \mathrm{GeV}$ the upper bound on the vector coupling is nearly the same for our three choices of $m_{R}$, as is the bound on total cross section. This is due to the fact that the transverse $R$ propagator becomes independent of $m_{R}$ once $\left(2 m_{\chi}\right)^{2} \gg m_{R}^{2}$. 

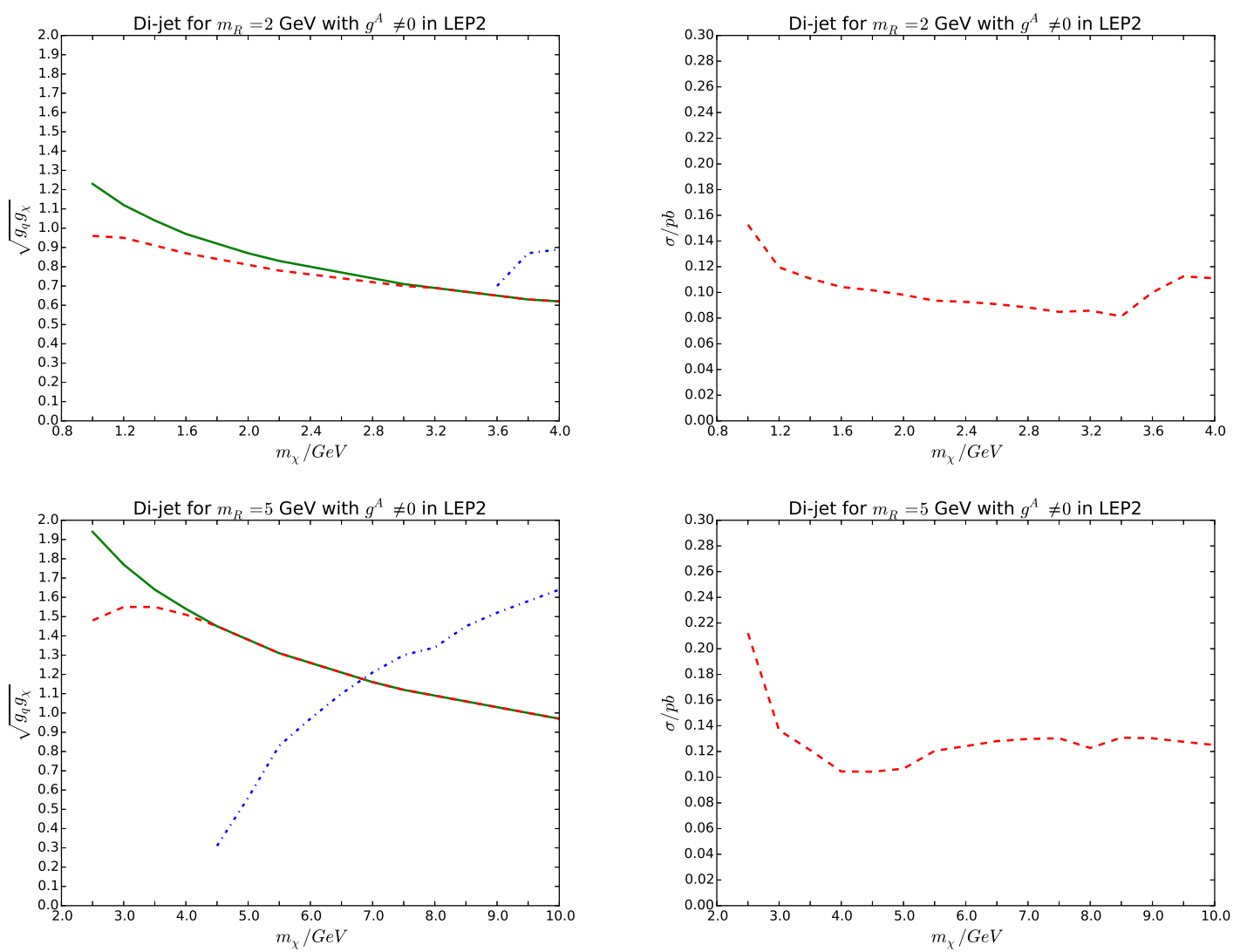

Figure 3. The meaning of the curves in the right frames, and of the solid and dashed curves in the left frames, is as in figure 2, but for mediator mass $m_{R}=2(5) \mathrm{GeV}$ in the top (bottom) frames. For $m_{\chi} \geq 3.6$ (4.5) GeV the solid and dashed curves coincide, i.e. the unitarity condition gives the stronger bound on the axial vector coupling. The dotted (blue) lines show the upper bound on the vector coupling that we derive from the ALEPH search, i.e. for these curves, $g_{q} g_{\chi}=g_{q}^{V} g_{\chi}^{V}$; the axial vector couplings were chosen such that the unitarity limit is saturated. In this mass range the upper bound on the signal cross section shown in the right frames also uses the maximal axial vector coupling allowed by unitarity.

In figures 2 and 3 we extended $m_{\chi}$ only to $m_{\chi}=2 m_{R}$. In figure 5 we show upper bounds on the couplings (left) and on the total cross section (right) for the same values of $m_{R}$, but for $m_{\chi}$ between 2 and $10 \mathrm{GeV}$. Moreover, we also compare the bounds on $g^{V}$ for $g^{A}=0$ (dotted curves) to the bounds on $g^{V}$ with $g^{A}$ chosen to saturate its upper bound (dashed curves), which is set by the unitarity condition (2.15) once $m_{\chi}>4 \mathrm{GeV}$. In this case $g_{\chi}^{A} g_{b}^{A} \propto m_{R}^{2} /\left(m_{\chi} m_{b}\right)$, so that the contribution from longitudinal $R$ exchange becomes largely independent of both $m_{\chi}$ and $m_{R}$ once $\left(2 m_{\chi}\right)^{2} \gg m_{R}^{2}$. Note that the axial vector coupling also contributes to the exchange of transverse $R$ bosons. This contribution simply scales like $\left(g^{A}\right)^{2}$, and is thus significant only for $m_{R}=5 \mathrm{GeV}$ where unitarity allows relatively large axial vector couplings. This explains why the upper bound on $g^{V}$ with maximal $g^{A}$ is stronger for $m_{R}=5 \mathrm{GeV}$ than for the smaller values of $m_{R}$. In contrast, if $g^{A}=0$ the bound on $g^{V}$ becomes independent of $m_{R}$ once $\left(2 m_{\chi}\right)^{2} \gg m_{R}^{2}$, as we saw 

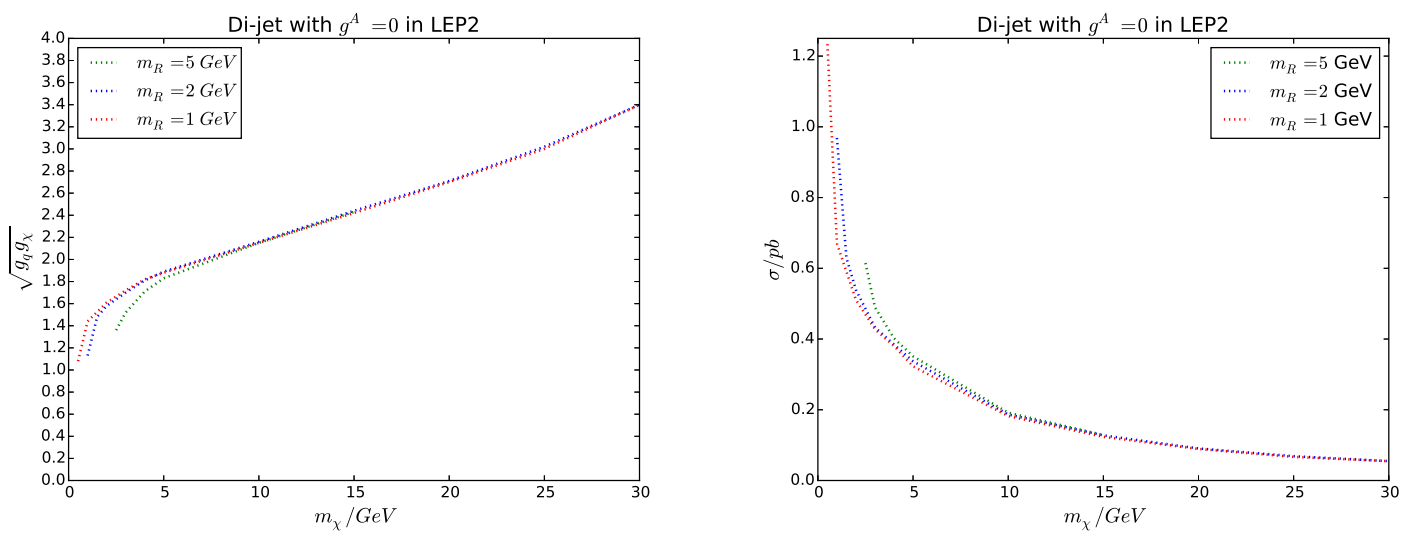

Figure 4. The left frame depicts the bound on $\sqrt{g_{q}^{V} g_{\chi}^{V}}$, while the right one shows the upper bound on the total signal cross section at $\sqrt{s}=208 \mathrm{GeV}$ from our recasting of the ALEPH limits; all axial vector couplings have been set to zero. The green, blue and red curves are for $m_{R}=5,2$ and $1 \mathrm{GeV}$, respectively. For $m_{\chi}>30 \mathrm{GeV}$ the bound on $g^{V}$ is weaker than the perturbativity condition (2.14).
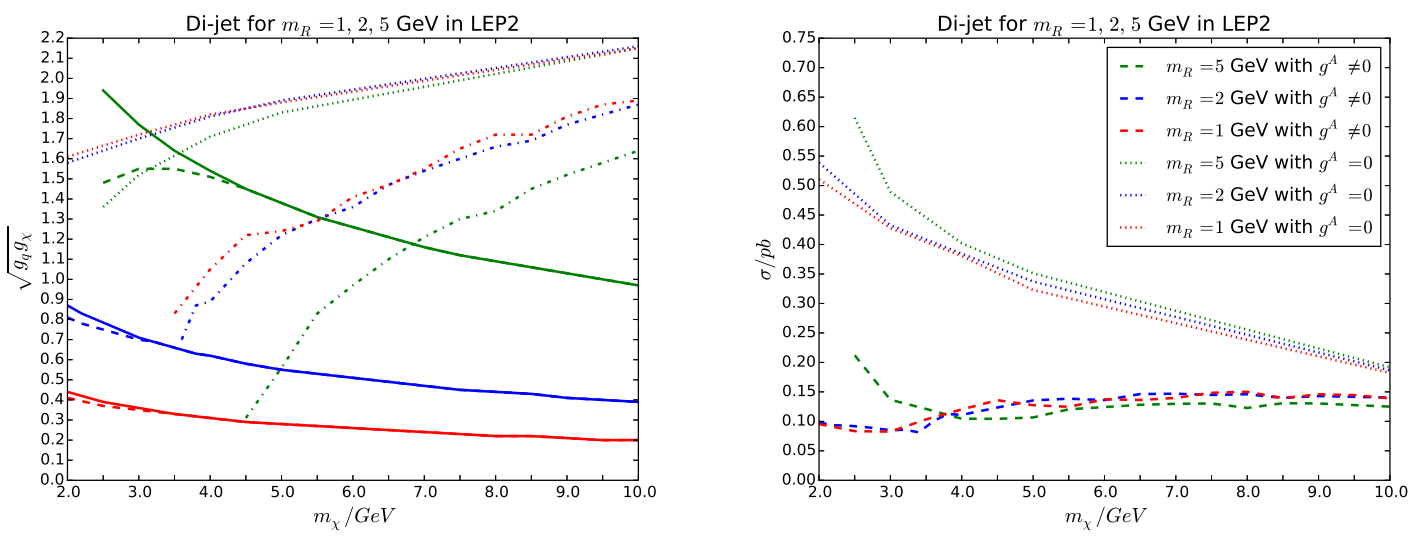

Figure 5. The left frame shows upper bounds on the couplings of the mediator. The solid lines are from the unitarity condition (2.15), while the dashed lines are from our recasting of the ALEPH squark search; both sets of curves refer to axial vector couplings. If the unitarity constraint is stronger, we allow non-vanishing vector couplings; their upper bounds, derived from the ALEPH data, are shown by the dot-dashed curves. If $g^{A}=0$, the unitarity condition are irrelevant, and the bounds on $g^{V}$ become weaker. They are shown by the dotted curves. The green, blue and red curves are for $m_{R}=5,2$ and $1 \mathrm{GeV}$, respectively. The right frame shows the corresponding upper bound on the total signal cross section before cuts at $\sqrt{s}=208 \mathrm{GeV}$. 
above. Overall figure 5 shows that the effect of $g^{A}$ can be significant even if it is much smaller than $g^{V}$.

The right frame of figure 5 again shows that the upper bound on the cross section becomes independent of $m_{R}$ once $\left(2 m_{\chi}\right)^{2} \gg m_{R}^{2}$. We also see that for light $R$ and $g^{A} \neq 0$, the upper bound on the cross section increases by nearly a factor of two once $m_{\chi}>4 \mathrm{GeV}$; evidently the cut efficiency becomes smaller. This coincides with the range of $\chi$ masses where the bound on the axial vector coupling is set by the unitarity constraint, so that the limit we derive from the ALEPH data can only be saturated by also including sizable vector couplings. The main observation is that the cut efficiency is much smaller if the process proceeds dominantly by vector coupling. For example, for $m_{R}=m_{\chi}=1 \mathrm{GeV}$, we find cut efficiencies between 1.5 and $3.5 \%$ for pure vector coupling, with couplings to $b$ quarks yielding the highest sensitivity. In contrast, if the cross section is dominated by the axial vector coupling to $b$ quarks the efficiency increases to $15 \%$. This is at least partly due to the fact that the $\chi \bar{\chi}$ pair has to be in a $P$ - wave in the $R$ rest frame if the $\chi \bar{\chi} R$ coupling is purely axial vector, whereas a vector coupling allows $S$-wave contributions. The $P$-wave has a larger $\chi \bar{\chi}$ invariant mass, making it easier to pass cuts related to the missing mass or missing energy. The cut efficiency increases with increasing $m_{\chi}$, which of course also implies larger $\chi \bar{\chi}$ invariant mass. However, even here pure vector couplings lead to lower cut efficiency. For example, for $m_{\chi}=10 \mathrm{GeV}$, i.e. at the end of the range shown in figure 5, we find a cut efficiency of just under $10 \%$ if $g^{A}$ saturates the unitarity bound, with little dependence on $m_{R}$; if $g^{A}=0$, the cut efficiency is only about $7.5 \%$.

We also find reduced cut efficiency if $m_{\chi}$ is only slightly above $m_{R} / 2$. In this case configurations where the $R$ boson is only slightly off-shell, i.e. configurations with small $\chi \bar{\chi}$ invariant mass, are even more strongly preferred dynamically than for larger values of the ratio $m_{\chi} / m_{R}$. This again leads to a reduced efficiency for cuts related to the missing mass.

\subsubsection{Analysis of LEP1 data}

Searches for the final state (3.1) were also performed at LEP1, the first period of operating the LEP collider (1989 to 1994), with $\sqrt{s} \simeq 91 \mathrm{GeV} \simeq m_{Z}[49,50]$. These analyses searched for $H \nu \bar{\nu}$ production where $H$ is the SM Higgs boson which is assumed to decay hadronically; this final state yielded the strongest lower bound on $m_{H}$ that could be derived from a single LEP1 analysis.

Since the exchanged $Z$ boson is now nearly on-shell, for not too large values of $m_{\chi}$ the total signal cross section is much larger than at LEP2. Moreover, the physics background at $\sqrt{s} \simeq m_{Z}$ is much smaller than at $\sqrt{s} \simeq 200 \mathrm{GeV}$. In particular, the $W^{+} W^{-}$and $Z Z$ backgrounds did not exist at LEP1. Therefore, less severe cuts were needed at LEP1, so the cut efficiency of our signal can be expected to be higher than for the LEP2 analyses. These two effects over-compensate the about three times smaller total luminosity accumulated at LEP1. At least for not too large DSP mass we therefore expect LEP1 data to lead to stronger constraints on the couplings of our model than LEP2 data.

In [49] the cuts and the number of selected events are not given in detail. We therefore cannot recast this analysis. Fortunately it is superseded by [50], where all applied cuts and 

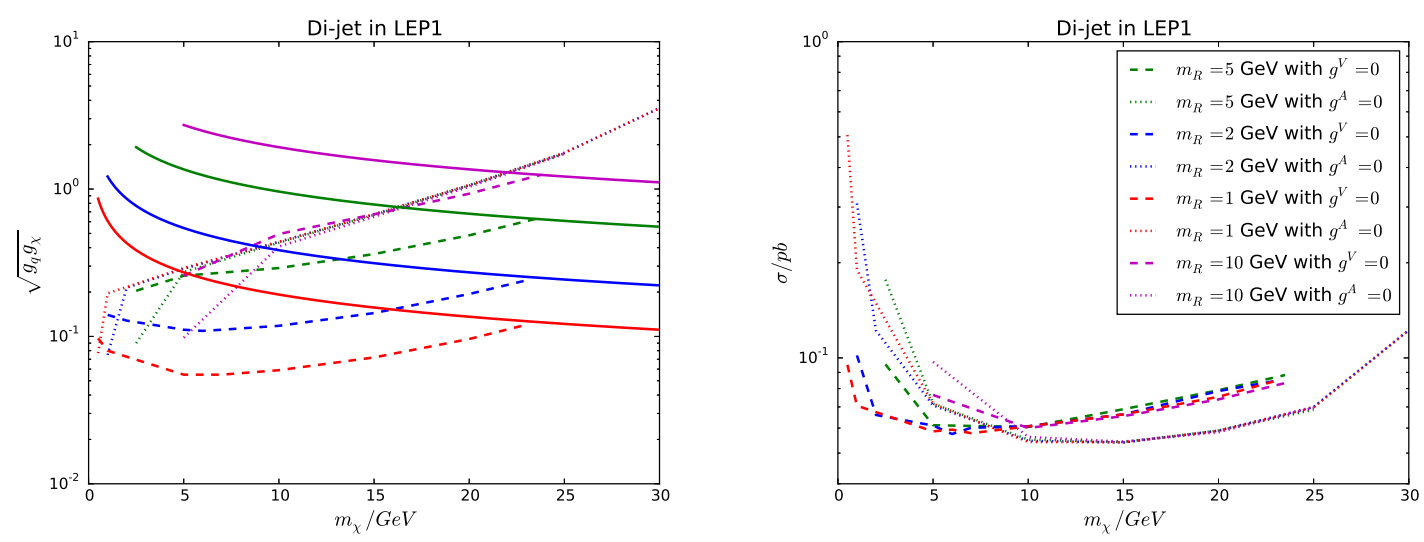

Figure 6. The left frame depicts bounds on couplings of the mediator. The solid lines show the unitarity bound on the axial vector coupling. The other curves depict bounds from our recasting of the ALEPH LEP1 limits. The dotted lines are the upper limits on $g^{V}$ for $g^{A}=0$, while the dashed lines are the upper limits on $g^{A}$ for $g^{V}=0$. The purple, green, blue and red curves are for $m_{R}=10,5,2$ and $1 \mathrm{GeV}$, respectively. For $m_{\chi}>30 \mathrm{GeV}$ the bound on $g^{V}$ is always weaker than the perturbativity condition (2.14). For $m_{\chi}>23 \mathrm{GeV}$ the LEP1 bound on $g^{A}$ is weaker than the unitarity condition (2.15), and is therefore not shown any more. The right frame shows the upper bound on the total signal cross section at $\sqrt{s}=91 \mathrm{GeV}$; we use the same conventions as in the left frame.

the number of selected events are listed. ${ }^{6}$ Unfortunately there is some uncertainty regarding the precise jet definition that has been used. One of the cuts requires to reconstruct the final state as exactly three jets. We found that the results differ slightly for different jet algorithms. Moreover, occasionally the reconstruction of the event as three-jet event does not work; we discard such events. However, both the effect of having to discard events that cannot be described as three-jet events, and the differences between final results using different jet algorithms, are quite small, probably smaller than the effects of ignoring detector smearing, as we do. In the results presented below we use the $k_{T}$ based Durham algorithm, which was the algorithm of choice for LEP2 analyses.

The results are shown in figure 6 . Evidently for $m_{\chi}<20 \mathrm{GeV}$ the bounds from LEP1 data are much better than those from the published analyses of LEP2 data. For larger DSP mass, however, the phase space constraints become quite severe at LEP1 energy, and hence these data quickly lose sensitivity. For pure axial vector coupling the upper bound on the coupling we derive from our recasting of the LEP1 data saturates the unitarity constraint at $m_{\chi} \simeq 23 \mathrm{GeV}$, with larger $m_{R}$ yielding a slightly larger range of $m_{\chi}$ where the experimental bound is below the unitarity limit. This can be understood as follows.

\footnotetext{
${ }^{6}$ The influential cuts are: $N_{\mathrm{ch}}>7, M_{\mathrm{vis}}<70 \mathrm{GeV}, p_{\mathrm{CH}} / \sqrt{s}>0.1, E_{30} / E_{\mathrm{vis}}>60 \%, E_{12}<3 \mathrm{GeV}$, $\theta_{\text {acol }}<165^{\circ}, M_{\text {vis }}>25 \mathrm{GeV}$ when $p_{T} / \sqrt{s}<10 \%, M_{\text {thrust }}^{1,2}>2.5 \mathrm{GeV}, \sum_{3 j} \theta_{j j}<342^{\circ}, \Phi_{\text {acop }}<159^{\circ}$, and $\Theta_{\text {miss }}^{\text {iso }}>31^{\circ}$. Here $p_{\mathrm{CH}}$ is the scalar sum of the charged particle momenta; $E_{30}$ is the energy measured at more than $30^{\circ}$ from the beam axis; $M_{\text {thrust }}^{1,2}$ is the invariant masses measured in both hemispheres according to the plane perpendicular to the thrust axis; and $\Theta_{\text {miss }}^{\text {iso }}$ is the largest cone around missing momentum vector containing energy less than $1 \mathrm{GeV}$. The other variables have already been defined in the LEP2 analysis described in section 3.1.1.
} 
The larger $m_{R}$, the larger the axial vector coupling allowed by unitarity. The contribution from longitudinal $R$ exchange is again independent of $m_{R}$ if the unitarity limit is saturated, but the contribution from the exchange of transversely polarized $R$ bosons increases with increasing $g^{A}$, and hence becomes significant only for larger $m_{R}$. Note also that for small $m_{R}$ the bound on $g^{A}$ at first becomes stronger as $m_{\chi}$ is increased from its minimal value, which we took to be just above $m_{R} / 2$ as before. As in figures 2 and 3 this is due to the exchange of longitudinal $R$ bosons.

In contrast, for $g^{A}=0$ the bounds on $g^{V}$ are strongest for the smallest value of $m_{\chi}$, where the $R$ boson only needs to be slightly off-shell. The steep rise of the dotted curves towards small $m_{\chi}$ in the right frame shows that the cut efficiency decreases, but this is over-compensated by the increase of the total cross section. Once $m_{\chi}>m_{R}$, the bound on $g^{V}$ again becomes largely independent of $m_{R}$, and is (coincidentally) quite close to the bound on $g^{A}$ for $m_{R}=10 \mathrm{GeV}$. The "experimental" upper bound on $g^{V}$ becomes worse than the perturbativity constraint $(2.14)$ once $m_{\chi}>30 \mathrm{GeV}$.

If $g^{A} \neq 0$ and $g^{V}=0$ the cut efficiency of our signal is generally higher than $20 \%$. For $5 \mathrm{GeV}<m_{\chi}<10 \mathrm{GeV}$ the cut efficiency is even higher than $30 \%$, and reaches the highest point of $32 \%$ for $m_{\chi}$ between $6 \mathrm{GeV}$ and $7 \mathrm{GeV}$. For $g^{V} \neq 0, g^{A}=0$ and relatively small $m_{\chi} \gtrsim m_{R} / 2$ the cut efficiency is again less, typically around $10 \%$, which is similar to the efficiency for the LEP2 squark pair search. However, it quickly increases for larger $m_{\chi}$, reaching $35 \%$ for $m_{\chi} \simeq 15 \mathrm{GeV}$. Moreover, for $m_{\chi}>10 \mathrm{GeV}$ the cut efficiency is now actually higher for pure vector coupling than for pure axial vector coupling. This is opposite to the results shown in figure 5 for LEP2 energies. The LEP1 analysis mostly employs cuts on angular variables, and does not contain any explicit cut on the invisible mass or energy; recall that such cuts play a prominent role in the corresponding analysis of LEP2 data.

\subsection{Four jet analysis}

We now turn to a discussion of the 4-jet final state. The signal again comes from the diagrams shown in figure 1 , except that the (real or virtual) $R$-boson now decays into a $q \bar{q}$ pair rather than a $\chi \bar{\chi}$ pair. As a result, at tree-level the cross section now only depends on the couplings of $R$ to quarks. We compute the signal by squaring the $R$-exchange contribution, i.e. we neglect interference between $R$-exchange and SM contributions. Note that the interference with the dominant (gluon exchange) SM contribution to the four quark final state is color suppressed $;^{7}$ moreover, the total SM contribution to four parton final states is dominated by $q \bar{q} g g$ production, where $g$ stands for a gluon.

There are several ALEPH analyses involving 4-jet final states. Some are optimized to detect $W^{+} W^{-}$or $Z Z$ final states. These are part of the background for us; hence these

\footnotetext{
${ }^{7}$ Denote the final state by $q\left(k_{1}\right) \bar{q}\left(k_{2}\right) q^{\prime}\left(k_{3}\right) \bar{q}^{\prime}\left(k_{4}\right)$, where $q^{\prime}$ may be a different flavor from $q$. The gluon exchange contribution where $q^{\prime}\left(k_{3}\right) \bar{q}^{\prime}\left(k_{4}\right)$ results from the splitting of a virtual gluon then only interferes with the $R$ exchange contributions where $q^{\prime}\left(k_{3}\right) \bar{q}\left(k_{2}\right)$ or $q\left(k_{1}\right) \bar{q}^{\prime}\left(k_{4}\right)$ originate from the decay of the $R$ boson. Evidently this is possible only if $q^{\prime}=q$, i.e. for final states with two identical $q \bar{q}$ pairs. Moreover, the interference gets a color factor of 1 , compared to a factor $N_{c}^{2}=9$ for the squared $R$ exchange diagram. We checked explicitly for some combinations of parameters that the interference terms change the total cross section only by a few percent.
} 

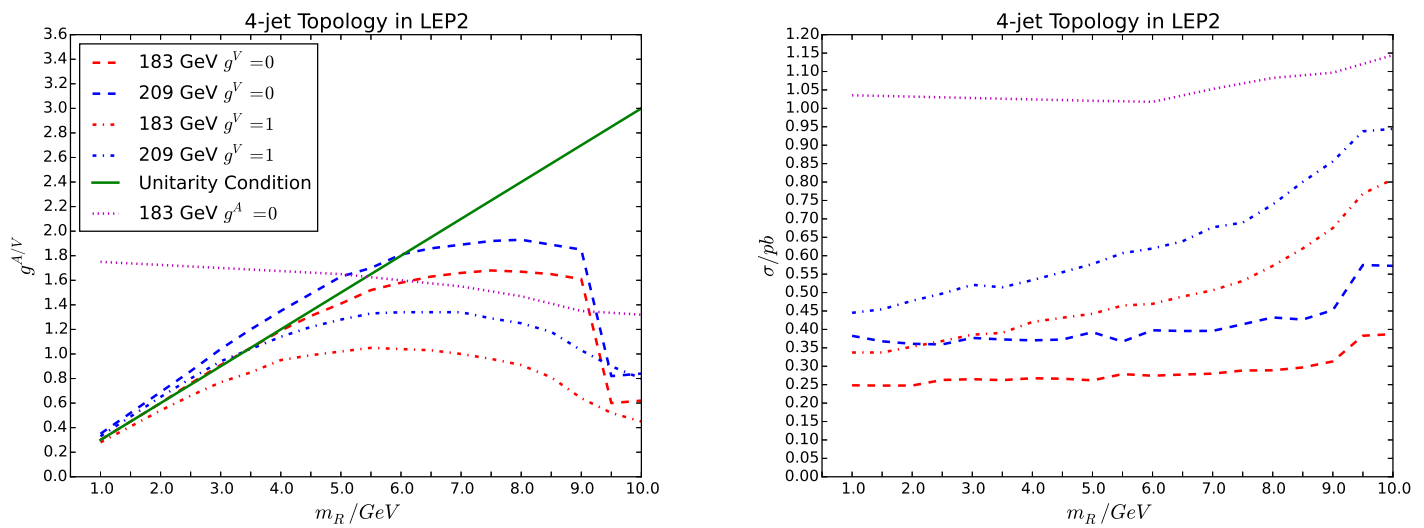

Figure 7. Bounds on the (axial) vector coupling to $b$ quarks (left) and total cross section (right) we derive from our recasting of the ALEPH Higgs searches in the 4-jet channel. In both frames the lower (red) curves correspond to data taken at $\sqrt{s}=183 \mathrm{GeV}$, while the upper (blue) curves correspond to data taken at $\sqrt{s}=209 \mathrm{GeV}$. The dashed curves have been obtained with vanishing vector couplings, while the dot-dashed curves are for $g_{q}^{V}=1$. The dotted magenta curves show the upper bounds for vanishing axial vector couplings. The solid (green) curve in the left frame shows the upper bound on $g_{b}^{A}$ from the unitarity constraint (2.15) applied to the $b$ quark.

analyses cannot be used to derive useful bounds on the couplings of our model. The earliest ALEPH analyses of the 4-jet final state in the LEP2 era had very low luminosity [51] or did not veto $Z Z$ events [52], and are hence also only of limited usefulness for our purpose.

In contrast, the searches for neutral Higgs bosons, either in pairs or in association with a $Z$ boson, investigate final states that are at least somewhat similar to ours. More importantly, they include cuts that attempt to minimize non-Higgs SM backgrounds, both from electroweak and from QCD sources. The related analyses cover the entire LEP2 energy range, from $\sqrt{s}=133$ to $209 \mathrm{GeV}$ [53-60]. The analyses of the data taken at $\sqrt{s} \leq 172 \mathrm{GeV}$ all use similar cuts, while the analyses of data taken at $\sqrt{s} \geq 183 \mathrm{GeV}$ apply another group of cuts in order to reduce $W^{+} W^{-}$and $Z Z$ backgrounds. The first group of analyses turns out to be essentially useless for us, due to the rather low energy and comparatively small integrated Luminosity.

However, the data taken at $\sqrt{s} \geq 183 \mathrm{GeV}$ do allow to impose meaningful constraints on our model. Although the cuts applied in these analyses are similar, the slight changes still influence the final efficiencies. We find the highest efficiency, of about $27 \%$ with little dependence on $m_{R}$, for the cuts applied to the data taken at $\sqrt{s}=183 \mathrm{GeV}[55],{ }^{8}$ where the $Z$ pair background is still very small. At the highest energy the efficiency falls to about 21 to $22 \%$. As a result, the strongest bound can be derived from the ALEPH analysis of the data taken at $\sqrt{s}=183 \mathrm{GeV}$. This is shown in figure 7 .

\footnotetext{
${ }^{8}$ The influential cuts are: at least $2 b$-jets, $N_{\mathrm{ch}}>7, \min \left(\cos \theta_{i j}+\cos \theta_{k l}\right)<-1.3(i j k l$ label the four jets), $\min \left(\sum_{i=1}^{4} \theta_{j j}^{i}\right)>350^{\circ}$, and either $y_{34}>(2.9-\# b-$ jets $) / 9.5$ (transition from 4 to 3 jets through Durham algorithm), or $m_{12}>78 \mathrm{GeV}, m_{34}>55 \mathrm{GeV}$, and $y_{34}>0.008$. Here $\theta_{i j}$ is the opening angle between jets $i$ and $j, m_{i j}$ is the invariant mass of the system of jets $i$ and $j ; \theta_{j j}^{i}$ is any one of these six opening angles, with the sum going over the smallest four; $\# b-$ jets is the number of tagged $b$-jets.
} 
The $183 \mathrm{GeV}$ analysis performs quite well. For pure axial vector coupling (dashed curves) the final cut efficiency for our signal is actually as good as the one for the allhadronic $Z H$ signal for which this analysis was originally designed. This leads to quite stringent bounds, in particular for small $m_{R}$, where it is significantly stronger than that from the 2-jet plus missing energy analysis of LEP2 data described in the previous section even for small $m_{\chi}$, if we assume $g_{q}=g_{\chi}$; of course, the constraints we derive from the analysis of the four jet final state are independent of $m_{\chi}$ and $g_{\chi}$, as long as $m_{\chi}>m_{R} / 2$. However, for vanishing vector couplings our "experimental" bound on $g_{b}^{A}$ is still slightly weaker than the one derived from the unitarity constraint (2.15) applied to the $b$ quark, where we used $m_{b}\left(m_{b}\right)=4.25 \mathrm{GeV}$.

Turning on a vector coupling $g_{q}^{V}=1$ for $q=s, c, b$ reduces the cut efficiency somewhat; this leads to increased upper bounds on the total cross section. This is presumably again due to the $P$-wave nature of the $q \bar{q}$ pair that originates from the "decay" of the virtual $R$ boson via an axial vector coupling, which leads to a larger separation between these two partons, and hence better separated jets. Nevertheless the resulting upper bound on $g_{b}^{A}$ that we derive from the $183 \mathrm{GeV}$ analysis is now better than the one from the unitarity condition. This is in particular true for larger $m_{R}$; the vector contribution depends less strongly on the mass of the mediator, since there are no terms $\propto m_{b}^{2} / m_{R}^{2}$ in this case. For small $m_{R}$ the upper bound on a pure vector coupling is rather weak, but still stronger than the perturbativity limit (2.14).

The bounds on the coupling become significantly stronger once on-shell $R \rightarrow b \bar{b}$ decays become possible. This region of larger $m_{R}$ is explored in figure 8. Since the unitarity bound becomes weaker for higher $m_{R}$, the final bound on the coupling is given by our recasting of the LEP2 search until $m_{R} \simeq 70 \mathrm{GeV}$, where it becomes comparable to the upper bound (2.14) from perturbativity. Over most of the range of $m_{R}$ shown, the curves for $g_{q}^{V}=0$ and $g_{q}^{V}=1$ behave similarly. Nevertheless, there are some differences for $m_{R}$ around 10 to $15 \mathrm{GeV}$. For pure axial vector coupling the bound on the coupling begins to rise again just after the point where on-shell $R \rightarrow b \bar{b}$ decays are allowed. In contrast, if the vector coupling is sizable, $g_{q}^{V}=1$, the lowest bound on the axial vector coupling is obtained for $m_{R} \simeq 12 \mathrm{GeV}$. The reason is that contributions due to the exchange of longitudinal $R$ bosons, which only comes from $g^{A}$, more strongly prefer small $m_{R}$. Hence turning on a vector coupling moves the peak of the cross section for fixed coupling to slightly larger values of $m_{R}$, where on-shell $R \rightarrow b \bar{b}$ decays are less phase space suppressed.

We also tried scenarios with pure vector coupling, setting $g_{b}^{A}=0$ (magenta dotted lines in figures 7 and 8). As in case of the 2 -jet plus missing energy analysis the resulting bound on the vector coupling is considerably weaker than that on $g^{A}$ for $m_{R}<5 \mathrm{GeV}$. This is partly due to the absence of the term enhanced by $m_{b}^{2} / m_{R}^{2}$, and partly due to the limited cut efficiencies, which are below $10 \%$ in this case. For $m_{R}<2 m_{b}$ the bound on the vector coupling does not depend much on $m_{R}$. The strongest bound appears for $m_{R} \gtrsim 2 m_{b}$. It gradually weakens again for larger $m_{R}$, saturating the perturbativity condition (2.14) around $m_{R}=35 \mathrm{GeV}$. In this case the cut efficiency is smaller than in the scenarios with non-vanishing $g^{A}$, as can be seen from the larger upper bound on the total signal cross section. 

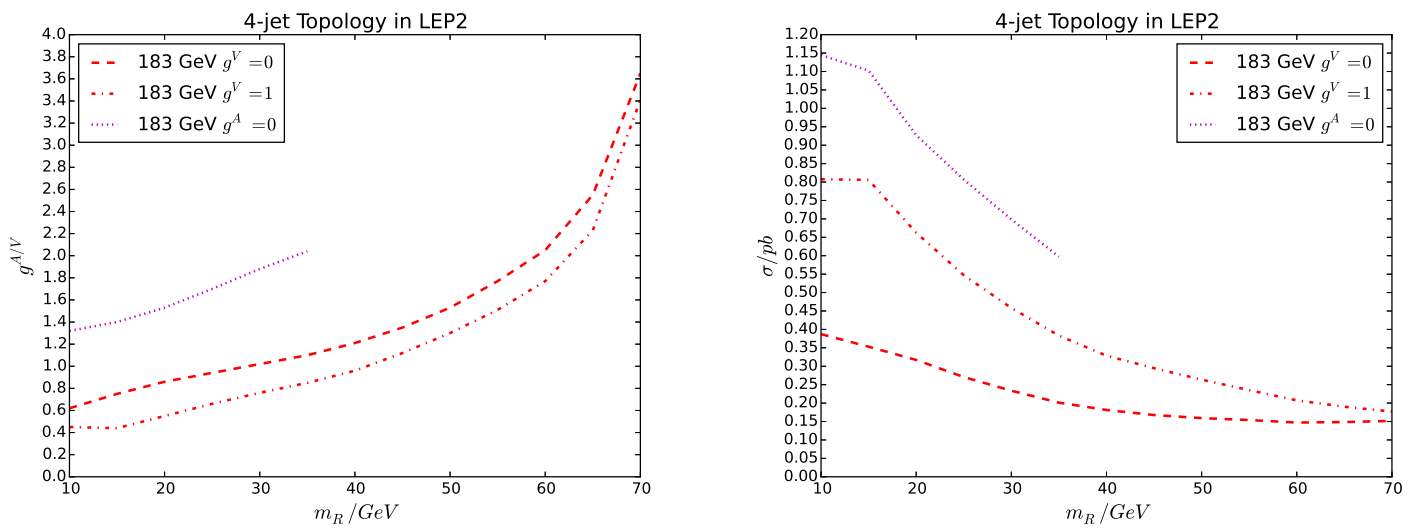

Figure 8. Upper bounds on the couplings (left) and total cross section (right) from our re-casting of an ALEPH LEP2 4-jet analysis. The notation is as in figure 7, except that we only show results for the analysis at $\sqrt{s}=183 \mathrm{GeV}$ which again has the highest sensitivity. For $g^{A}=0$, shown by the dotted curve, the LEP2 bound is only stronger than the perturbativity condition (2.14) if $m_{R}<35 \mathrm{GeV}$. The unitarity bound on $g_{b}^{A}$ becomes weaker for higher $m_{R}$, and is no longer relevant. However, for $m_{\chi}>70 \mathrm{GeV}$ the perturbativity condition is stronger than our "experimental" bound, both for $g_{q}^{V}=0$ and for $g_{q}^{V}=1$.

We saw that in case of the two jet plus missing $E_{T}$ analysis the strongest bound often comes from LEP1 data rather from LEP2 data, due to the larger signal cross section (for not too large $m_{\chi}$ ) and smaller SM background. Unfortunately the only published ALEPH new physics searches at LEP1 using four jet final states $[49,61]$ are based on early data samples with very low integrated luminosity of $1.16 / \mathrm{pb}$. These searches were designed for the pair production of particles with sizable coupling to the $Z$ boson, e.g. light charged Higgs bosons, with each new particle decaying into a pair of jets. The early data set was sufficient to exclude such particles with mass nearly up to $M_{Z} / 2$; at the time there was thus little motivation to perform new physics searches in the four jet final state using the full LEP1 data sample. Even in the absence of backgrounds the early analyses could improve on the bounds we derive from the LEP2 searches only if the cut efficiency of signal events was higher than $50 \%$; this is even higher than the efficiency of the final states for which these searches were originally optimized. We conclude that among the published four jet analyses, the one based on data taken at $\sqrt{s}=183 \mathrm{GeV}$ data gives the tightest constraints on our model.

\section{Improved analysis of LEP2 data}

In section 3 we saw that the cuts applied in the searches for 2-jet plus missing energy searches in LEP2 analysis published by the ALEPH collaboration have limited efficiency for our signal, below $5 \%$ in some cases, which is obviously not satisfactory. In this section we therefore propose new cuts, which have much better efficiency for our signal but still remove most SM backgrounds. We employed kinematical variables also used by ALEPH, and applied them to SM events generated with Pythia 8.2 [36]. The cuts are designed to 


\begin{tabular}{|c|c|}
\hline \multicolumn{2}{|c|}{ Double Jet + Missing Energy $(j j \not p)$} \\
\hline Variable & Selection Rule \\
\hline$M_{\mathrm{vis}}$ & $>4 \mathrm{GeV}$ \\
\hline$p_{T} / E_{\mathrm{vis}}$ & $>20 \%$ \\
\hline$\left|\cos \theta_{\text {miss }}\right|$ & $<0.95$ \\
\hline$\Delta \Phi_{T}$ & $<170^{\circ}$ \\
\hline$E_{\mathrm{NH}}$ & $<30 \% E_{v i s}$ \\
\hline$(\mathrm{NH}=$ Neutral Hadron $)$ & \begin{tabular}{l|l}
$30 \% \leqslant E_{\text {vis }}<45 \%$ & $p_{T(\mathrm{NH})}>1.8 \% \sqrt{s}$ \\
\end{tabular} \\
\hline$E_{l_{1}} / \sqrt{s}$ & $<10 \%$ \\
\hline$E_{12} / \sqrt{s}$ & $<5 \%$ \\
\hline$E_{l_{1}}^{30} / \sqrt{s}$ & $>1 \%$ \\
\hline Durham & $M_{j_{1}} \leqslant 9 M_{j_{2}}$ and $M_{j_{2}} \leqslant 9 M_{j_{1}}$ \\
\hline 2 -jet $j_{1} j_{2}$ & $M_{j_{1} j_{2}} \leqslant 80 \mathrm{GeV}$ or $M_{j_{1} j_{2}} \geqslant 100 \mathrm{GeV}$ \\
\hline$y_{23}$ & $<0.02$ \\
\hline
\end{tabular}

Table 1. Cuts designed to reduce the SM background to the two jet plus missing $E_{T}$ signal. The cuts listed above the last horizontal double line are mostly directed against two-photon events, whereas the cuts below this double line help to remove background events containing on-shell $W$ or $Z$ bosons. See the text for further details.

remove all SM backgrounds that may appear for the energy level up to $208 \mathrm{GeV}$. When analyzing data taken below the $Z$ pair threshold part of the cuts can be loosened or removed, which would increase the cut efficiency even further. As the cut efficiency of the 4-jet signature as applied to our model is already as good as that of the $Z H$ search for which this analysis was originally optimized, we only try to redesign the selection rules for the $j j \not p$ signature.

We start by requiring at least 5 good tracks in the event; this essentially guarantees that the event contains some hadronic activity.

Most multi-hadron events produced by LEP2 were not due to $e^{+} e^{-}$annihilation. Rather, they were produced when both the electron and the positron emitted a spacelike photon; these two photons then collided to produce a multi-hadronic final state. Since the virtuality of these photons can be of order of the electron mass, they can be considered to be quasi-real. This class of events is therefore often called two-photon (or $\gamma \gamma$ ) events. The first set of cuts, listed above the second double line in table 1, are designed to greatly reduce this background. These cuts are adapted from the cuts against two-photon events employed in ref. [39]; we use the same kinematical variables, but change some of the cut values since we optimize our analysis for higher energies, $\sqrt{s}=208 \mathrm{GeV}$.

The first of these cuts requires the invariant mass of the system consisting of all detected particles to exceed $4 \mathrm{GeV}$. Since the probability for the emission of a nearly on-shell photon off an energetic electron or positron quickly increases with decreasing photon energy, the $\gamma \gamma$ background peaks at small values of this variable. 
The second cut imposes a lower bound on the total transverse momentum of the system of visible particles. Since photons are mostly emitted with small virtuality, the $\gamma \gamma$ system typically has small total transverse momentum. It is nonzero partly due to measurement errors, and partly because the detector is not hermetic. In particular, an outgoing $e^{ \pm}$ can carry some transverse momentum but still escape detection. It is important to note that this cut also removes $e^{+} e^{-} \rightarrow q \bar{q}$ annihilation events (including events with additional gluon emission), since here the total visible transverse momentum is also close to zero.

The third cut vetoes events where the missing momentum vector, which is simply the opposite of the total 3-momentum of all detected particles, points nearly in the forward or backward reaction. There is no reason why the two quasi-real photons should have similar energy. If the outgoing $e^{ \pm}$remain undetected, two-photon events therefore typically have a large longitudinal momentum of detected particles, i.e. the total missing momentum vector is dominated by its longitudinal component.

The fourth cut employs the transverse acoplanarity angle $\Delta \Phi_{T}$ defined in [38]. It removes events where the momenta in two hemispheres are nearly back-to-back. This cut is again efficient against both two-photon and $e^{+} e^{-} \rightarrow q \bar{q}$ annihilation events.

The next cut, which we again copy from ALEPH analyses, uses the energy $E_{\mathrm{NH}}$ carried by neutral hadrons. It can be measured by subtracting the energy associated with the tracks of charged particles from the total energy measured in the calorimeters. All events where $E_{\mathrm{NH}}$ is less than $30 \%$ of $E_{\mathrm{vis}}$ pass this cut. Events where $E_{\mathrm{NH}}>0.45 E_{\mathrm{vis}}$ are always removed. If $E_{\mathrm{NH}}$ lies between these two values, events only pass if the visible $p_{T}$ not including neutral hadrons, called $p_{T \mathrm{NH}}$ in the table, is at least $0.018 \sqrt{s}$. The purpose of this combination of cuts is to remove events where a large fraction of the energy, or of the transverse momentum, is assigned to neutral hadrons. This can be dangerous, since the energies and momenta of neutral hadrons are least well determined experimentally of all "visible" particles (i.e., not counting neutrinos or DSPs); hence these events may contain a large amount of "fake" missing (transverse) energy, due to mismeasurement of the neutral hadrons.

The penultimate cut in this category vetoes events with energetic charged leptons (electrons or muons); $l_{1}$ is the most energetic identified charged lepton in the event. This removes two-photon events where at least one of the photons is so far off-shell that the corresponding outgoing $e^{ \pm}$becomes detectable. This cut will also be effective against other backgrounds, in particular against events with leptonically decaying $W$ bosons; these events are dangerous since they also contain a neutrino, which leads to an imbalance of the visible (transverse) momentum. Of course, events that do not contain a charged lepton also pass this cut.

The last cut against two-photon events removes events where the energy $E_{12}$ deposited in forward or backward direction (within $12^{\circ}$ of the beam axis) exceeds $0.05 \sqrt{s}$. Note that two-photon events can have a sizable visible energy, even if the transverse momentum is typically small. This cut also removes events where one of the outgoing $e^{ \pm}$hits the detector, but is not identified as a charged lepton.

The second group of cuts mostly targets events with real $W$ or $Z$ bosons. The first of these uses the variable $E_{l_{1}}^{30}$, which is the energy of particles in a $30^{\circ}$ half-angle cone around the most energetic charged lepton (excluding the lepton itself). This cut is applied only 


\begin{tabular}{|c|c|c|c|c|}
\hline \multicolumn{5}{|c|}{$m_{R}=5 \mathrm{GeV}$} \\
\hline$m_{\chi} / \mathrm{GeV}$ & 2.5 & 3.0 & 3.5 & 4.0 \\
\hline$\epsilon_{A}$ & $18.48 \%$ & $23.53 \%$ & $27.54 \%$ & $29.22 \%$ \\
\hline$\epsilon_{V}$ & $12.32 \%$ & $15.55 \%$ & $18.09 \%$ & $19.83 \%$ \\
\hline$m_{\chi} / \mathrm{GeV}$ & 4.5 & 5 & 5.5 & 6.0 \\
\hline$\epsilon_{A}$ & $30.74 \%$ & $32.21 \%$ & $33.42 \%$ & $33.58 \%$ \\
\hline$\epsilon_{V}$ & $21.43 \%$ & $22.53 \%$ & $22.97 \%$ & $23.52 \%$ \\
\hline$m_{\chi} / \mathrm{GeV}$ & 6.5 & 7.0 & 7.5 & 8.0 \\
\hline$\epsilon_{A}$ & $34.46 \%$ & $35.02 \%$ & $34.76 \%$ & $35.57 \%$ \\
\hline$\epsilon_{V}$ & $25.94 \%$ & $25.44 \%$ & $26.14 \%$ & $27.25 \%$ \\
\hline$m_{\chi} / \mathrm{GeV}$ & 8.5 & 9.0 & 9.5 & 10.0 \\
\hline$\epsilon_{A}$ & $35.51 \%$ & $35.45 \%$ & $36.15 \%$ & $36.25 \%$ \\
\hline$\epsilon_{V}$ & $27.29 \%$ & $27.26 \%$ & $28.37 \%$ & $29.43 \%$ \\
\hline
\end{tabular}

Table 2. Cut Efficiencies for $m_{R}=5 \mathrm{GeV}$ and $m_{R} / 2 \leq m_{\chi} \leq 2 m_{R} . \epsilon_{A}$ has been computed with pure axial vector coupling, $g_{q}^{V}=g_{\chi}^{V}=0$, while $\epsilon_{V}$ is the efficiency for pure vector coupling, assumed to be the same for $s, c$ and $b$ quarks, while $g_{\chi}^{A}=g_{q}^{A}=0$.

if the event contains such a lepton. It removes events where this lepton is isolated, which is typically the case for leptons from leptonic $W^{ \pm}$decays. In contrast, charged leptons produced in the decay of $c$ or $b$ quarks typically have a lot of hadronic activity nearby, i.e. large values of $E_{l_{1}}^{30}$, and thus pass this cut.

The three final cuts concern the jet system. In order to apply these cuts, the event is forced into a two-jet topology using the Durham $k_{T}$ algorithm. The first cut removes events where one jet is very "slim", i.e. has very small invariant mass. This is often the case for a jet from a hadronically decaying $\tau$ lepton. This cut thus removes events containing real $W^{ \pm} \rightarrow \tau^{ \pm} \nu_{\tau}$ decays. The second cut removes events where the di-jet invariant mass is close to $M_{Z}$; this removes $Z Z$ events with one $Z$ boson decaying hadronically and the other into a neutrino pair, i.e. invisibly. The last cut removes events where the event would be reconstructed as containing three or more jets for dimensionless resolution variable $y_{23}=0.02$. We find that this cut removes very efficiently that part of the $e \nu_{e} W$ background that survived the lepton cuts.

Some resulting cut efficiencies are listed in tables 2 to 4 . We focus on scenarios with rather light mediator and light DSP, where the efficiency of our signal for the published missing energy searches at LEP2, discussed in the previous section, was especially poor. For $m_{R}=5 \mathrm{GeV}$, table 2 , we show efficiencies for pure vector and pure axial vector couplings separately; for $m_{R}=2 \mathrm{GeV}$, table 3 , and $m_{R}=1 \mathrm{GeV}$, table 4 , we only show results for pure axial vector coupling, since outside the region $m_{\chi} \simeq m_{R} / 2$ the cut efficiency for pure vector coupling has very little dependence on $m_{R}$.

We see that the efficiency for pure vector coupling quickly increases from $m_{\chi}=m_{R} / 2$ to $m_{\chi} \simeq m_{R}$, and then gradually increase to $35 \%$ for $m_{\chi}>25 \mathrm{GeV}$. These efficiencies 


\begin{tabular}{|c|c|c|c|c|}
\hline \multicolumn{5}{|c|}{$m_{R}=2 \mathrm{GeV}$} \\
\hline$m_{\chi} / \mathrm{GeV}$ & 1.0 & 1.2 & 1.4 & 1.6 \\
\hline$\epsilon$ & $21.98 \%$ & $25.82 \%$ & $27.59 \%$ & $29.30 \%$ \\
\hline$m_{\chi} / \mathrm{GeV}$ & 1.8 & 2.0 & 2.2 & 2.4 \\
\hline$\epsilon$ & $29.25 \%$ & $30.91 \%$ & $31.08 \%$ & $31.65 \%$ \\
\hline$m_{\chi} / \mathrm{GeV}$ & 2.6 & 2.8 & 3.0 & 3.2 \\
\hline$\epsilon$ & $32.07 \%$ & $32.86 \%$ & $33.36 \%$ & $33.65 \%$ \\
\hline$m_{\chi} / \mathrm{GeV}$ & 3.4 & 3.6 & 3.8 & 4.0 \\
\hline$\epsilon$ & $33.25 \%$ & $33.40 \%$ & $34.29 \%$ & $34.49 \%$ \\
\hline
\end{tabular}

Table 3. Cut Efficiencies for $m_{R}=2 \mathrm{GeV}$ and $m_{R} / 2 \leq m_{\chi} \leq 2 m_{R}$. We have assumed pure axial vector coupling, $g_{q}^{V}=g_{\chi}^{V}=0$.

\begin{tabular}{|c|c|c|c|c|}
\hline \multicolumn{5}{|c|}{$m_{R}=1 \mathrm{GeV}$} \\
\hline$m_{\chi} / \mathrm{GeV}$ & 0.5 & 0.6 & 0.7 & 0.8 \\
\hline$\epsilon$ & $24.36 \%$ & $27.36 \%$ & $28.99 \%$ & $28.57 \%$ \\
\hline$m_{\chi} / \mathrm{GeV}$ & 0.9 & 1.0 & 1.1 & 1.2 \\
\hline$\epsilon$ & $28.77 \%$ & $29.49 \%$ & $30.71 \%$ & $30.23 \%$ \\
\hline$m_{\chi} / \mathrm{GeV}$ & 1.3 & 1.4 & 1.5 & 1.6 \\
\hline$\epsilon$ & $30.85 \%$ & $30.82 \%$ & $31.43 \%$ & $30.22 \%$ \\
\hline$m_{\chi} / \mathrm{GeV}$ & 1.7 & 1.8 & 1.9 & 2.0 \\
\hline$\epsilon$ & $31.98 \%$ & $31.19 \%$ & $32.38 \%$ & $31.25 \%$ \\
\hline
\end{tabular}

Table 4. Cut Efficiencies for $m_{R}=1 \mathrm{GeV}$ and $m_{R} / 2 \leq m_{\chi} \leq 2 m_{R}$. We have assumed pure axial vector coupling, $g_{q}^{V}=g_{\chi}^{V}=0$.

are about three times higher than those for the published analysis discussed in the previous section.

Turning to axial vector couplings, the cut efficiency for any combination $\left(m_{R}, m_{\chi}\right)$ is again better than the corresponding one in the published analysis described in the previous section. For example, for $m_{R}=2 m_{\chi}$, the efficiency is more than three times larger. As in case of vector couplings, the cut efficiency quickly increases when $m_{\chi}$ is raised from $m_{R} / 2$ to $m_{R}$; it continues to increase more slowly for even higher $m_{\chi}$, reaching slightly more than $40 \%$ for $m_{\chi}>30 \mathrm{GeV}$. Cut efficiencies of 30 to $40 \%$ are quite typical for many LEP searches.

The selection cuts were chosen to remove most SM backgrounds. We simulated $\gamma \gamma$ (i.e., $\left.e^{+} e^{-} \rightarrow e^{+} e^{-} q \bar{q}\right)$ events; events with hadronically decaying $W^{+} W^{-}$or $Z Z$ pairs leading to events with four hard partons prior to showering; $Z \bar{\nu} \nu, Z l^{+} l^{-}$and $W l \nu_{l}$ events where the gauge boson decays hadronically; and $e^{+} e^{-} \rightarrow q \bar{q}$ annihilation events. We include "purely hadronic" final states since they can contain heavy $b$ or $c$ quarks whose semileptonic decays can produce energetic neutrinos, and hence lead to significant amounts of missing energy. 
The $Z \bar{\nu} \nu, Z l^{+} l^{-}$and $W l \nu_{l}$ events include contributions where the lepton pair comes from the decay of a (nearly) on-shell $Z$ or $W$ boson, but also contributions that only arise at third order in electroweak couplings. The latter diagrams do not contribute very much to the total cross sections for these final state, but populate different regions of phase space.

Our cuts remove more than $99.9 \%$ of most of these SM backgrounds. The exceptions are the $W l \nu_{l}$ and $Z \bar{\nu} \nu$ final states, where $1.05 \%$ and $5.03 \%$, respectively, of all generated events pass the cuts. MadGraph finds total cross sections of $7.34 \mathrm{pb}$ and $0.33 \mathrm{pb}$, respectively, for these two final states, leading to a total SM background of about $0.1 \mathrm{pb}$. Recall that the upper bounds on the signal cross section we derived in the previous section, shown in the right frames of figures $2-5$, were $\gtrsim 0.1 \mathrm{pb}$.

For parameter choices that saturate these earlier bounds, the new cuts would therefore lead to comparable signal and background cross sections. Since we cannot apply the new cuts to the actual data, we cannot quote the resulting bounds, even if the cut efficiencies are roughly doubled over a broad range of parameters. In order to give some idea of the expected improvement, we give some sensitivity limits, i.e. expected bounds (computed under the assumption that the observed number of events agrees exactly with the SM prediction). To this end, we use the $p$-value test of the "null" hypothesis (SM only) for a $95 \%$ confidence level. For $m_{R}=5 \mathrm{GeV}$ with $g^{V}=0$, the upper limit on $g^{A}$ is improved from 1.48 to 1.39 at $m_{\chi}=2.5 \mathrm{GeV}$, and the point that LEP data is weaker than unitarity condition (2.15) moves from $m_{\chi}=4.5 \mathrm{GeV}$ to $5 \mathrm{GeV}$. The sensitivity to the vector couplings increases even more. For $m_{R}=5 \mathrm{GeV}$ and $m_{\chi}=2.5 \mathrm{GeV}$ with $g^{A}=0$, the bound of $g^{V}$ is improved from 1.36 to 0.99 . For $m_{R}=5 \mathrm{GeV}$ and $m_{\chi}=10 \mathrm{GeV}$, the expected bound on $g^{V}$ is improved from 2.15 to 1.88 with $g^{A}=0$, and from 1.64 to 1.53 with non-zero $g^{A}$ reaching unitarity bound. We repeat that actual bounds can only be derived by applying our cuts to real data.

Further optimization of the cuts, in order to maximize $S / B$ or $S / \sqrt{B}$ where $S$ is the signal and $B$ is the background, should be possible. For example, the (dominant) $W l \nu_{l}$ background can be further reduced by slightly reducing the lower end of the excluded region of the invariant mass of the di-jet system (the penultimate cut in table 1). However, such an optimization should also include detector effects, which is difficult for us to do reliably. This analysis nevertheless makes it appear likely that the bounds we derived in the previous section, which used published analyses not optimized for this final state, can be improved significantly.

\section{$5 \quad$ Summary and conclusions}

This study derives constraints from published ALEPH searches, based on data taken at the LEP collider some twenty years ago, on a simplified dark matter model. The model features a fermionic dark sector particle (DSP $\chi$ ) and a spin-1 mediator $R$ which has sizable couplings to some quarks but not to leptons. A complete model may contain additional Higgs bosons to generate $m_{R}$ and/or additional fermions for anomaly cancellation (see e.g. [62]), but the presence of these particles should not affect our interpretation of LEP data. This kind of simplified model has of course been analyzed previously, in particular 
in connection with LHC data, which impose severe constraints from "monojet" searches if $m_{R}>2 m_{\chi}$, and from searches for di-jet resonances for heavy $R$. We therefore focus on rather light mediators, $m_{R} \lesssim 70 \mathrm{GeV}$, and always require $m_{R}<2 m_{\chi}$ so that on-shell $R \rightarrow \chi \bar{\chi}$ decays are kinematically forbidden. We also impose unitarity and perturbativity constraints on the parameters of the model.

We consider two different final states. The new physics production of two jets plus missing energy and momentum, $j j \not p$, can only proceed via off-shell $R$ exchange; the signal is thus proportional to the square of the product of the mediator's coupling to quarks and to the DSP. In contrast, in our model the production of 4 -jet final states can occur through real or virtual $R$ exchange, and the signal depends only on the mediator's coupling to quarks. We used ALEPH data since this experiment published analyses of both of these final states, including complete descriptions of the applied cuts and numbers of surviving SM background events. This allowed us to recast these analyses; although we did not implement detector effects, these are likely to be less important for the signal than for the background (where they can e.g. create missing momentum).

The best bound on the $j$ jpp final state from LEP2 data (taken at $\sqrt{s}$ well above the $Z$ mass) comes from squark searches. Somewhat counter-intuitively the resulting bound on the couplings becomes stronger for larger $m_{\chi}$ if $R$ is very light and axial vector couplings dominate. This is partly because increasing $m_{\chi}$ increases the cut efficiency, since it increases the kinematical lower bound on the missing energy in the event; however, the main effect is the increase of the contribution from longitudinal $R$ bosons, whose matrix element scales like $g_{\chi}^{A} g_{b}^{A} m_{\chi} m_{b} / m_{R}^{2}$. However, even though this is the most promising among several ALEPH searches for this kind of final state, the cut efficiency for our model is rather low, less than $20 \%$. In particular, for vanishing axial vector couplings the bound on the vector coupling is worse than that from perturbativity. In section 4 where therefore devised an optimized set of cuts, which according to our simulation still removes most SM backgrounds, but has significantly higher efficiency for $q \bar{q} \chi \bar{\chi}$ events in our model.

For $m_{\chi} \lesssim 20 \mathrm{GeV}$ the best bounds nevertheless come from LEP1 data, taken at $\sqrt{s} \simeq$ $M_{Z}$, well below the $W^{+} W^{-}$and $Z Z$ production thresholds. We found that an ALEPH analysis looking for $\nu \bar{\nu} H$ final states, where $H$ is the SM Higgs boson which is assumed to decay hadronically, uses cuts that have quite a high efficiency to $q \bar{q} \chi \bar{\chi}$ events in our model. For example, for $m_{R}=1 \mathrm{GeV}$ and $m_{\chi} \lesssim 20 \mathrm{GeV}$ it requires $\sqrt{g_{b}^{A} g_{\chi}^{A}} \leq 0.1$, see figure 6 . However, LEP1 data cannot probe the region $m_{\chi} \gtrsim 25 \mathrm{GeV}$ for couplings that respect the unitarity and perturbativity constraints.

Turning to the four jet final state, we found that ALEPH searches for $Z H$ production in the all-hadronic final state have quite a good cut efficiency for $q \bar{q} q^{\prime} \bar{q}^{\prime}$ production via real or virtual $R$ exchange in our model. The resulting bound on the coupling of the mediator are roughly comparable to those that follow from $j j \not p$ final states at LEP2, if the DSP is light and the mediator couples with equal strength to quarks and to the DSP. This search allows to exclude new parts of parameter space for $m_{R} \leq 70 \mathrm{GeV}$. For somewhat smaller $m_{R}$ we again expect LEP1 data to be considerably more sensitive, due to the larger signal cross section and reduced background. Unfortunately the only published ALEPH analysis 
of four jet final states at LEP1 used only about $1 \%$ of the total integrated luminosity. This was sufficient to exclude the pair production of new particles with masses up to nearly the beam energy, which was the purpose of this search, but does not allow to improve the limits we derive from LEP2 data.

In all cases we found that the Dirac structure of the couplings (vector or axial vector) affects the bounds significantly. This is partly due to enhanced contributions from longitudinal $R$ exchange, which are proportional to axial vector couplings. Moreover, the cut efficiencies often differ, with pure axial vector couplings usually leading to higher efficiency; the exception is the di-jet plus missing energy search at LEP1, where for $m_{\chi}>10 \mathrm{GeV}$ vector couplings lead to a higher cut efficiency.

In summary, we have shown that LEP data should be able to impose significant new constraints on the parameter space of dark matter models with a leptophobic spin-1 mediator, if the mass of the mediator and/or the dark matter particle are in the (tens of) $\mathrm{GeV}$ range and on-shell decays of the mediator into the dark matter particles are forbidden. While a published LEP1 search for di-jet plus missing energy final states already has good efficiency for our model, even the best published analysis of the same final state using LEP2 data has quite a low efficiency. Conversely, the best LEP2 analysis of four jet final states is already quite useful for our purposes, but published LEP1 searches for this final state only use a small fraction of all data. Improved analyses of LEP data therefore hold considerable promise to probe new regions of parameter space of this class of models.

\section{Acknowledgments}

This work was partially supported by the SFB TR33 funded by the Deutsch Forschungsgemeinschaft, and partially by the by the German ministry for scientific research (BMBF).

Open Access. This article is distributed under the terms of the Creative Commons Attribution License (CC-BY 4.0), which permits any use, distribution and reproduction in any medium, provided the original author(s) and source are credited.

\section{References}

[1] J.L. Feng, Dark Matter Candidates from Particle Physics and Methods of Detection, Ann. Rev. Astron. Astrophys. 48 (2010) 495 [arXiv: 1003.0904] [INSPIRE].

[2] S. Banerjee, S. Matsumoto, K. Mukaida and Y.-L.S. Tsai, WIMP Dark Matter in a Well-Tempered Regime: A case study on Singlet-Doublets Fermionic WIMP, JHEP 11 (2016) 070 [arXiv : 1603.07387] [INSPIRE].

[3] M. Hoferichter, P. Klos, J. Menéndez and A. Schwenk, Improved limits for Higgs-portal dark matter from LHC searches, Phys. Rev. Lett. 119 (2017) 181803 [arXiv:1708.02245] [INSPIRE].

[4] GAMBIT collaboration, P. Athron et al., Status of the scalar singlet dark matter model, Eur. Phys. J. C 77 (2017) 568 [arXiv:1705.07931] [InSPIRE]. 
[5] A. DiFranzo, K.I. Nagao, A. Rajaraman and T.M.P. Tait, Simplified Models for Dark Matter Interacting with Quarks, JHEP 11 (2013) 014 [Erratum ibid. 01 (2014) 162] [arXiv: 1308.2679] [INSPIRE].

[6] J. Abdallah et al., Simplified Models for Dark Matter Searches at the LHC, Phys. Dark Univ. 9-10 (2015) 8.

[7] G. Busoni et al., Recommendations on presenting LHC searches for missing transverse energy signals using simplified s-channel models of dark matter, arXiv:1603.04156 [INSPIRE].

[8] ATLAS collaboration, Search for dark matter and other new phenomena in events with an energetic jet and large missing transverse momentum using the ATLAS detector, JHEP 01 (2018) 126 [arXiv:1711.03301] [INSPIRE].

[9] CMS collaboration, Search for dark matter produced with an energetic jet or a hadronically decaying $W$ or $Z$ boson at $\sqrt{s}=13 \mathrm{TeV}$, JHEP 07 (2017) 014 [arXiv: 1703. 01651] [INSPIRE].

[10] CMS collaboration, Search for dijet resonances in proton-proton collisions at $\sqrt{s}=13 \mathrm{TeV}$ and constraints on dark matter and other models, Phys. Lett. B 769 (2017) 520 [Erratum ibid. B 772 (2017) 882] [arXiv: 1611.03568] [INSPIRE].

[11] ATLAS collaboration, Search for new phenomena in dijet events using $37 \mathrm{fb}^{-1}$ of $p p$ collision data collected at $\sqrt{s}=13 \mathrm{TeV}$ with the ATLAS detector, Phys. Rev. D 96 (2017) 052004 [arXiv: 1703.09127] [INSPIRE].

[12] ATLAS collaboration, Search for low-mass dijet resonances using trigger-level jets with the ATLAS detector in pp collisions at $\sqrt{s}=13$ TeV, Phys. Rev. Lett. 121 (2018) 081801 [arXiv: 1804.03496] [INSPIRE].

[13] ATLAS and CMS collaborations, M. Bauce, Search for new physics in dijet final states in ATLAS and CMS, in 5th Large Hadron Collider Physics Conference (LHCP 2017) Shanghai, China, May 15-20, 2017, arXiv:1709.04754 [INSPIRE].

[14] K.S. Babu, C.F. Kolda and J. March-Russell, Implications of generalized Z-Z' mixing, Phys. Rev. D 57 (1998) 6788 [hep-ph/9710441] [InSPIRE].

[15] E. Dudas, Y. Mambrini, S. Pokorski and A. Romagnoni, (In)visible $Z^{\prime}$ and dark matter, JHEP 08 (2009) 014 [arXiv:0904.1745] [INSPIRE].

[16] E. Accomando, A. Belyaev, L. Fedeli, S.F. King and C. Shepherd-Themistocleous, $Z^{\prime}$ physics with early LHC data, Phys. Rev. D 83 (2011) 075012 [arXiv: 1010.6058] [INSPIRE].

[17] P.J. Fox, J. Liu, D. Tucker-Smith and N. Weiner, An Effective Z', Phys. Rev. D 84 (2011) 115006 [arXiv: 1104.4127 ] [INSPIRE].

[18] M.T. Frandsen, F. Kahlhoefer, S. Sarkar and K. Schmidt-Hoberg, Direct detection of dark matter in models with a light $Z^{\prime}$, JHEP 09 (2011) 128 [arXiv:1107.2118] [INSPIRE].

[19] A. Alves, S. Profumo and F.S. Queiroz, The dark $Z^{\prime}$ portal: direct, indirect and collider searches, JHEP 04 (2014) 063 [arXiv:1312.5281] [INSPIRE].

[20] G. Jungman, M. Kamionkowski and K. Griest, Supersymmetric dark matter, Phys. Rept. 267 (1996) 195 [hep-ph/9506380] [INSPIRE].

[21] GAMBIT collaboration, A. Kvellestad, First SUSY results with GAMBIT, PoS (EPS-HEP2017) 299 [arXiv: 1710. 02503] [INSPIRE].

[22] E. Bagnaschi et al., Likelihood Analysis of the pMSSM11 in Light of LHC 13-TeV Data, Eur. Phys. J. C 78 (2018) 256 [arXiv:1710.11091] [InSPIRE]. 
[23] M.T. Frandsen, F. Kahlhoefer, A. Preston, S. Sarkar and K. Schmidt-Hoberg, LHC and Tevatron Bounds on the Dark Matter Direct Detection Cross-Section for Vector Mediators, JHEP 07 (2012) 123 [arXiv: 1204.3839] [INSPIRE].

[24] E.W. Kolb and M.S. Turner, The Early Universe, Front. Phys. 69 (1990) 1 [inSPIRE].

[25] F. Kahlhoefer, K. Schmidt-Hoberg, T. Schwetz and S. Vogl, Implications of unitarity and gauge invariance for simplified dark matter models, JHEP 02 (2016) 016 [arXiv: 1510.02110] [INSPIRE].

[26] ALEPH collaboration, D. Decamp et al., ALEPH: A detector for electron-positron annnihilations at LEP, Nucl. Instrum. Meth. A 294 (1990) 121 [Erratum ibid. A 303 (1991) 393] [INSPIRE].

[27] ALEPH collaboration, D. Buskulic et al., Performance of the ALEPH detector at LEP, Nucl. Instrum. Meth. A 360 (1995) 481 [INSPIRE].

[28] J. Alwall, M. Herquet, F. Maltoni, O. Mattelaer and T. Stelzer, MadGraph 5: Going Beyond, JHEP 06 (2011) 128 [arXiv:1106.0522] [INSPIRE].

[29] M. Chala, F. Kahlhoefer, M. McCullough, G. Nardini and K. Schmidt-Hoberg, Constraining Dark Sectors with Monojets and Dijets, JHEP 07 (2015) 089 [arXiv: 1503.05916] [INSPIRE].

[30] Particle Data Group collaboration, C. Patrignani et al., Review of Particle Physics, Chin. Phys. C 40 (2016) 100001 [inSPIRE].

[31] PandaX-II collaboration, A. Tan et al., Dark Matter Results from First 98.7 Days of Data from the PandaX-II Experiment, Phys. Rev. Lett. 117 (2016) 121303 [arXiv:1607.07400] [INSPIRE].

[32] CRESST collaboration, G. Angloher et al., Results on light dark matter particles with a low-threshold CRESST-II detector, Eur. Phys. J. C 76 (2016) 25 [arXiv:1509.01515] [INSPIRE].

[33] PICO collaboration, C. Amole et al., Dark Matter Search Results from the PICO-60 $C_{3} F_{8}$ Bubble Chamber, Phys. Rev. Lett. 118 (2017) 251301 [arXiv:1702.07666] [INSPIRE].

[34] A. Alloul, N.D. Christensen, C. Degrande, C. Duhr and B. Fuks, FeynRules 2.0 - A complete toolbox for tree-level phenomenology, Comput. Phys. Commun. 185 (2014) 2250 [arXiv: 1310.1921] [INSPIRE].

[35] C. Degrande, C. Duhr, B. Fuks, D. Grellscheid, O. Mattelaer and T. Reiter, UFO - The Universal FeynRules Output, Comput. Phys. Commun. 183 (2012) 1201 [arXiv:1108.2040] [INSPIRE].

[36] T. Sjöstrand et al., An Introduction to PYTHIA 8.2, Comput. Phys. Commun. 191 (2015) 159 [arXiv:1410.3012] [INSPIRE].

[37] ALEPH collaboration, R. Barate et al., Searches for sleptons and squarks in $e^{+} e^{-}$collisions at $189 \mathrm{GeV}$, Phys. Lett. B 469 (1999) 303 [INSPIRE].

[38] ALEPH collaboration, A. Heister et al., Search for stable hadronizing squarks and gluinos in $e^{+} e^{-}$collisions up to $\sqrt{s}=209$ GeV, Eur. Phys. J. C 31 (2003) 327 [hep-ex/0305071] [INSPIRE].

[39] ALEPH collaboration, R. Barate et al., Searches for charginos and neutralinos in $e^{+} e^{-}$ collisions at $\sqrt{s}=161 \mathrm{GeV}$ and $172 \mathrm{GeV}$, Eur. Phys. J. C 2 (1998) 417 [hep-ex/9710012] [INSPIRE]. 
[40] ALEPH collaboration, R. Barate et al., Search for charginos and neutralinos in $e^{+} e^{-}$ collisions at center-of-mass energies near $183 \mathrm{GeV}$ and constraints on the MSSM parameter space, Eur. Phys. J. C 11 (1999) 193 [INSPIRE].

[41] ALEPH collaboration, R. Barate et al., Search for supersymmetric particles in $e^{+} e^{-}$ collisions at $\sqrt{s}$ up to $202 \mathrm{GeV}$ and mass limit for the lightest neutralino, Phys. Lett. B 499 (2001) 67 [hep-ex/0011047] [INSPIRE].

[42] OPAL collaboration, G. Abbiendi et al., Search for chargino and neutralino production at $\sqrt{s}=189 \mathrm{GeV}$ at LEP, Eur. Phys. J. C 14 (2000) 187 [Erratum ibid. C 16 (2000) 707] [hep-ex/9909051] [INSPIRE].

[43] ALEPH collaboration, A. Heister et al., Absolute mass lower limit for the lightest neutralino of the MSSM from $e^{+} e^{-}$data at $\sqrt{s}$ up to $209 \mathrm{GeV}$, Phys. Lett. B 583 (2004) 247 [InSPIRE].

[44] ALEPH collaboration, R. Barate et al., Search for invisible Higgs boson decays in $e^{+} e^{-}$ collisions at center-of-mass energies up to $184 \mathrm{GeV}$, Phys. Lett. B 450 (1999) 301 [INSPIRE].

[45] L3 collaboration, M. Acciarri et al., Missing mass spectra in hadronic events from $e^{+} e^{-}$ collisions at $\sqrt{s}=161 \mathrm{GeV}-172 \mathrm{GeV}$ and limits on invisible Higgs decay, Phys. Lett. B 418 (1998) 389 [INSPIRE].

[46] ALEPH collaboration, R. Barate et al., Search for an invisibly decaying Higgs boson in $e^{+} e^{-}$ collisions at $189 \mathrm{GeV}$, Phys. Lett. B 466 (1999) 50 [INSPIRE].

[47] P.J. Fox, R. Harnik, J. Kopp and Y. Tsai, Missing Energy Signatures of Dark Matter at the LHC, Phys. Rev. D 85 (2012) 056011 [arXiv:1109.4398] [INSPIRE].

[48] CMS collaboration, Search for dark matter, extra dimensions and unparticles in monojet events in proton-proton collisions at $\sqrt{s}=8 \mathrm{TeV}$, Eur. Phys. J. C 75 (2015) 235 [arXiv: 1408.3583] [INSPIRE].

[49] ALEPH collaboration, D. Decamp et al., Searches for new particles in $Z$ decays using the ALEPH detector, Phys. Rept. 216 (1992) 253 [INSPIRE].

[50] ALEPH collaboration, D. Buskulic et al., Mass limit for the standard model Higgs boson with the full LEP-1 ALEPH data sample, Phys. Lett. B 384 (1996) 427 [INSPIRE].

[51] ALEPH collaboration, D. Buskulic et al., Four jet final state production in $e^{+} e^{-}$collisions at center-of-mass energies of $130 \mathrm{GeV}$ and $136 \mathrm{GeV}, \mathrm{Z}$. Phys. C 71 (1996) 179 [INSPIRE].

[52] ALEPH collaboration, R. Barate et al., Four jet final state production in $e^{+} e^{-}$collisions at center-of-mass energies ranging from $130 \mathrm{GeV}$ to $184 \mathrm{GeV}$, Phys. Lett. B 420 (1998) 196 [INSPIRE].

[53] ALEPH collaboration, R. Barate et al., Search for the neutral Higgs bosons of the MSSM in $e^{+} e^{-}$collisions at $\sqrt{s}$ from $130 \mathrm{GeV}$ to $172 \mathrm{GeV}$, Phys. Lett. B 412 (1997) 173 [INSPIRE].

[54] ALEPH collaboration, R. Barate et al., Search for the Standard Model Higgs boson in $e^{+} e^{-}$ collisions at $\sqrt{s}=161 \mathrm{GeV}, 170 \mathrm{GeV}$ and $172 \mathrm{GeV}$, Phys. Lett. B 412 (1997) 155 [INSPIRE].

[55] ALEPH collaboration, R. Barate et al., Search for the standard model Higgs boson at the LEP-2 collider near $\sqrt{s}=183$ GeV, Phys. Lett. B 440 (1998) 403 [hep-ex/9811032] [INSPIRE].

[56] ALEPH collaboration, R. Barate et al., Search for the neutral Higgs bosons of the standard model and the MSSM in $e^{+} e^{-}$collisions at $\sqrt{s}=189 \mathrm{GeV}$, Eur. Phys. J. C 17 (2000) 223 [INSPIRE]. 
[57] ALEPH collaboration, J. Nielsen, Search for neutral Higgs bosons in $e^{+} e^{-}$collisions at $\sqrt{s}$ less than $=196 \mathrm{GeV}$, in Lepton and photon interactions at high energies. Proceedings, 19th International Symposium, LP'99, Stanford, U.S.A., August 9-14, 1999, hep-ex/9908016 [INSPIRE].

[58] ALEPH collaboration, R. Barate et al., Searches for neutral Higgs bosons in $e^{+} e^{-}$collisions at center-of-mass energies from $192 \mathrm{GeV}$ to $202 \mathrm{GeV}$, Phys. Lett. B 499 (2001) 53 [hep-ex/0010062] [INSPIRE].

[59] ALEPH collaboration, R. Barate et al., Observation of an excess in the search for the standard model Higgs boson at ALEPH, Phys. Lett. B 495 (2000) 1 [hep-ex/0011045] [INSPIRE].

[60] ALEPH collaboration, A. Heister et al., Final results of the searches for neutral Higgs bosons in $e^{+} e^{-}$collisions at $\sqrt{s}$ up to $209 \mathrm{GeV}$, Phys. Lett. B 526 (2002) 191 [hep-ex/0201014] [INSPIRE].

[61] ALEPH collaboration, D. Decamp et al., Search for the neutral Higgs bosons of the MSSM and other two doublet models, Phys. Lett. B 265 (1991) 475 [INSPIRE].

[62] M. Duerr and P. Fileviez Perez, Theory for Baryon Number and Dark Matter at the LHC, Phys. Rev. D 91 (2015) 095001 [arXiv:1409.8165] [InSPIRE]. 